Japan. J. Math.

Vol. 14, No. 1, 1988

\title{
Triangular matrix representation for selfadjoint operators in Krein spaces
}

\author{
By Yoshiomi Nakagami and Minoru Tomita
}

(Received May 12, 1986)

\section{§1. Introduction}

We shall consider an indefinite inner product space which is written in the form of a direct sum of two Hilbert spaces and the sign of whose inner product is negative on either one of the spaces. Usually, such a space is called a Krein space. Since it has the original Hilbertian inner product as well as the indefinite inner product, it seems to be the most manageable space among indefinite inner product spaces. For operators in a Krein space we can consider two adjoint operations: $x \rightarrow x^{*}$ and $x \rightarrow x^{\sharp}$ with respect to the Hilbertian inner product and the indefinite inner product, respectively. Using the \#-operation, we can introduce the concepts of \#-selfadjoint, \#-unitary, \#-projection, \#-positive, \#-negative and so forth in the same way as in a Hilbert space.

It is a special feature of a Hilbert space among linear topological vector spaces that we can develop naturally the spectral analysis for operators. The analogous structures are also expected in a Krein space. Indeed, many mathematicians and physisists have been making efforts to develop such a theory in the last half of this century, as shown in References. However a standard technique is not established yet.

Now, we shall explain the circumstances in a Krein space more closely. First the spectrum of a \#-selfadjoint operator is symmetric with respect to the real line and is distributed to the outside of the line. Moreover there exists a \#-selfadjoint operator whose spectrum is the whole complex plane. The dimension of the eigenspace corresponding to the nonreal spectrum is less than twice the smaller of the dimensions of the two spaces. Secondly, a \#-selfadjoint operator is not necessarily diagonalizable even in a finite dimensional space. We can represent it in a standard form similar to the Jordan normal form. However, at present we have no idea how to extend the Jordan normal form to an infinite dimensional space. Therefore, as for a normal or standard form we are required to find a completely new idea and a new method. 
Notwithstanding the situation, the number of people who are interested in the indefinite inner product spaces are not small. The main reason for this may be that not only does the structure of an indefinite inner product space appear naturally in various fields such as differential equations, real analysis, field theory, etc., but also we get the feeling that favorable results can be anticipated for them.

A Krein space is called a Pontrjagin space, if either of the Hilbert spaces defining the Krein space is finite dimensional. Some of the properties on a Krein space become simpler on a Pontrjagin space. For instance unlike in a general Krein space a \#-unitary is automatically bounded in a Pontrjagin space. In [15] one of the authors has proposed a new treatment of a \#-selfadjoint operator on a Pontrjagin space: if a \#-selfadjoint operator $h$ is bounded, then it is represented in the form of an upper triangular $3 \times 3$ matrix $\left(h_{i j}\right)$ of operators such that $h_{11}$ and $h_{33}$ act on neutral closed subspaces and $h_{22}$ is \#-spectral, namely, it is similar to a selfadjoint operator with respect to a \#-unitary. The main purpose of this paper is to study to what extent we can extend the above result to the cases where the \#-selfadjoint operator is unbounded or the space is a general Krein space. Immediately we encounter problems on the selfadjointness and the product of unbounded operators. In order to treat these difficulties we must consider two cases depending on whether the \#-unitary implementing the similarity is bounded or not. In these cases the \#-selfadjoint operator is called \#-spectral or quasi-\#-spectral, respectively. If a \#-selfadjoint operator is quasi-\#-spectral, then it is reconstructed from the similar selfadjoint operator together with the \#-unitary implementing the similarity. If it is \#-spectral, it is also selfadjoint for some Hilbertian inner product. Thus the \#-spectrality preserves the spectrum.

In Section 2 we will explain the technical terms and some known results on a Krein space. In order to extend the above $3 \times 3$ matrix representation to the one in a Krein space, in Section 3 we shall assume the boundedness of the operator and the compactness of the off diagonal components of $2 \times 2$ matrix representation of the operator with respect to the two Hilbert spaces defining the Krein space. Then we can prove our desired result, in which the $(2,2)$ component is quasi-\#-spectral. The crucial point in our discussion is to show that the symmetric operator similar to the given one is selfadjoint. While the latter assumption stated above is needed only for applying the idea used in [12], it seems to be essential for our statement. As a next step we want to remove the boundedness of the operator by using the Cayley transforms. For this we will prepare in Section 4 a result to the effort that the Cayley transform preserves the compactness of the off diagonal components under some assumptions. In Section 5 we will show our desired result for an unbounded \#-selfadjoint operator in a Pontrjagin space by choosing a 
Hilbertian inner product suitably. We will also give an example which indicates that the method of Cayley transforms does not work in general in a Krein space. In Section 6 we will apply the results obtained in the above to a one parameter group of bounded \#-unitaries and obtain an upper triangular $3 \times 3$ matrix representation. For an indecomposable one Araki has given a physical interpretation as a Gupta-Bleuler triplet in [3]. In the last Section 7 we will give three examples concerning the quasi-\#-spectrality.

This paper gives a detailed proof for some of the statements made in [10, $16]$.

\section{§2. Preliminary}

In this paper we will use the traditional terminologies and notations in the theory of Hibert spaces.

Definition 2.1. An inner product space $\{\Re,\langle\rangle$,$\} on C$ is called a Krein space if there exists a positive definite inner product $(1)$ with respect to which $\Re$ is a Hilbert space and $\langle$,$\rangle is of the form$

$$
\langle\xi, \eta\rangle=(J \xi \mid \eta), \quad \xi, \eta \in \Re
$$

for some selfadjoint unitary $J$.

We will also use the notation $\{\Re, J\}$ for a Krein space. For a complex vector space with an indefinite inner product $\langle$,$\rangle , it is necessary and$ sufficient for a Hilbertian inner product $(\mid)$ to be selfdual i.e. $\|\xi\|=$ $\sup _{\|\eta\| \leq 1}|\langle\xi, \eta\rangle|$, that the selfadjoint operator $J$ defined by (2.1) is unitary. The operator $J$ in Definition 2.1 is called a metric operator. Since it is selfadjoint unitary, it is decomposed into the difference $J^{+}-J^{-}$of two projections $J^{ \pm}$ with $J^{+}+J^{-}=1$. Denote the ranges of $J^{ \pm}$by $\Re^{ \pm}$.

A vector $\xi$ in $\Re$ is called \#-positive, neutral or \#-negative according as $\langle\xi, \xi\rangle$ is positive, zero or negative. The same terminologies will be used for subspaces and for projections. Moreover, a subspace $\mathfrak{M}$ is called uniformly \#-positive or uniformly \#-negative if there exists a positive constant $\alpha$ such that $\langle\xi, \xi\rangle \geq \alpha\|\xi\|^{2}$ or $\langle\xi, \xi\rangle \leq-\alpha\|\xi\|^{2}$ on $\mathfrak{M}$, respectively.

The adjoint $x^{\#}$ of a densely defined linear operator $x$ with respect to the indefinite inner product is defined similarly as the case of a Hilbert space:

$$
\langle x \xi, \eta\rangle=\left\langle\xi, x^{\sharp} \eta\right\rangle, \quad \xi \in D(x), \quad \eta \in D\left(x^{\sharp}\right) .
$$

Then $D\left(x^{\sharp}\right)=J D\left(x^{*}\right)$ and $x^{\sharp}=J x^{*} J$. A densely defined operator $x$ satisfying $x=x \#$ is said to be \#-selfadjoint. A \#-selfadjoint operator defined everywhere is called a $\#$-projection if it is idempotent. A \#-projection is automatically 
bounded and has a closed range. A closed subspace $\mathfrak{M}$ is uniformly \#-positive (resp. uniformly \#-negative) if and only if there exists a \#-positive (resp. \#negative) \#-projection onto $\mathfrak{M}$. A densely defined operator $v$ is called a \#unitary if it has a densely defined inverse and $v^{\#}=v^{-1}$. An operator $v$ is a bounded \#-unitary if and only if $v^{\sharp} v=v v^{\sharp}=1$.

When $\Re^{+}$or $\Re^{-}$is finite dimensional, the Krein space is sometimes called a Pontrjagin space. The fact that a Krein space is a Pontrjagin space simplifies the situation extremely. The typical example is given by \#-unitary operators. These operators are automatically bounded in a Pontrjagin space, unlike in a Krein space which is not a Pontrjagin space.

Definition 2.2 ([15, 12]). A \#-selfadjoint (resp. \#-unitary) operator $x$ is called \#-spectral if there exists a bounded \#-unitary $v$ such that $v x v^{\#}$ is selfadjoint (resp. unitary).

Definition 2.3 ([12]). A \#-selfadjoint (resp. \#-unitary) operator $x$ is called quasi-\#-spectral if there exists a \#-unitary $v$ such that

(i) $D\left(v^{*} v\right) \subset D(x)$ and $x D\left(v^{*} v\right) \subset D\left(v^{*} v\right)$ (resp. $\left.x D\left(v^{*} v\right)=D\left(v^{*} v\right)\right)$,

(ii) the restriction of $v x v^{-1}$ to $D_{0}$ is closable and the closure is selfadjoint (resp. unitary), where $D_{0}=v D\left(v^{*} v\right)$.

In Definition 2.3, if $v$ is bounded, then $x$ is automatically bounded. Thus the definition is too restrictive to be applied to unbounded \#-selfadjoint operators in a Pontrjagin space. In Section 6 we will also use the same terminologies used in Definitions 2.2 and 2.3 for a one parameter group of bounded \#-unitaries.

REMARK (a) $D_{0}$ is dense in $\Re$.

(b) The \#-unitary $v$ is bounded if and only if $D(|v|)=R(|v|)$. Indeed, let $p_{1}, p_{2}$ and $p_{3}$ be the spectral projections of $|v|$ corresponding to the intervals $(0,1),\{1\}$ and $(1, \infty)$, respectively. Since $|v|$ is \#-selfadjoint, it satisfies $J|v| J$ $=|v|^{-1}$ and hence $J p_{1} J=p_{3}$ and $J p_{2} J=p_{2}$. Thus $D(|v|)=D\left(|v|^{-1}\right)$ if and only if both $|v| p_{3}$ and $|v|^{-1} p_{1}$ are bounded, if and only if $|v|$ is bounded.

(c) From condition (i) in Definition 2.3 we obtain $R\left(v^{*} v\right) \subset D\left(x^{*}\right)$ and $x^{*} R\left(v^{*} v\right) \subset R\left(v^{*} v\right)$ (resp. $x^{*} R\left(v^{*} v\right)=R\left(v^{*} v\right)$ ).

(d) From condition (ii), the closure of $v x v^{\#} \mid D_{0}$ is also \#-selfadjoint (resp. \#-unitary).

(e) If $x$ is a bounded \#-selfadjoint (resp. \#-unitary) operator which is quasi-\#-spectral with respect to a $\#$-unitary $v$, then the closure $y$ of $v x v^{\#} \mid D_{0}$ is selfadjoint (resp. unitary). Conversely, the closure of $v^{\sharp} y v \mid D\left(v^{*} v\right)$ coincides with the original $x$. Thus $x$ is reconstructed from $y$ and $v$.

Proposition 2.4. Let $v$ be $a \#$-unitary and let $\AA_{v}$ be the completion of 
$D\left(v^{*} v\right)$ with respect to the inner product $(\mid)_{v}$ defined by $(\xi \mid \eta)_{v}=(v \xi \mid v \eta)$. If $x$ is a bounded \#-selfadjoint (resp. \#-unitary) operator in $\Re$ with (i) in Definition 2.3 , then $x$ is quasi-\#-spectral if and only if the closure of $x \mid D\left(v^{*} v\right)$ is selfadjoint (resp. unitary) in $\Re_{v}$.

Proof. First we suppose that $x$ is a bounded \#-selfadjoint operator. For a \#-unitary $v$, if $\xi, \eta \in D\left(v^{*} v\right)$, then

$$
(x \xi \mid \eta)_{v}=\left(\left(v x v^{-1}\right) v \xi \mid v \eta\right)
$$

and

$$
(\xi \mid x \eta)_{v}=\left(v \xi \mid\left(v x v^{-1}\right) v \eta\right) .
$$

Thus the restriction of $x$ to $D\left(v^{*} v\right)$ is symmetric in $\AA_{v}$ if and only if the restriction of $v x v^{\#}$ to $D_{0}$ is symmetric in $\AA$. Therefore $v x v^{\sharp} \mid D_{0}$ is essentially selfadjoint, namely $v(x \pm i 1) D\left(v^{*} v\right)=\left(v x v^{\sharp} \pm i 1\right) D_{0}$ is dense in $\AA$, if and only if $(x \pm i 1) D\left(v^{*} v\right)$ is dense in $\Re_{v}$. Consequently, $x$ is quasi-\#-spectral if and only if $x \mid D\left(v^{*} v\right)$ is essentially selfadjoint in $\AA_{v}$.

Now, suppose that $x$ is a bounded $\#$-unitary. Then for any $\xi, \eta \in D\left(v^{*} v\right)$ we have

$$
(x \xi \mid x \eta)_{v}=\left(\left(v x v^{\sharp}\right) v \xi \mid\left(v x v^{\sharp}\right) v \eta\right)
$$

and

$$
(\xi \mid \eta)_{v}=(v \xi \mid v \eta)
$$

Since $x$ and $v x v^{\#}$ have dense domains and dense ranges, we see that the closure of $v x v^{\sharp} \mid D_{0}$ is unitary in $\Re$, if and only if $x$ is unitary in $\AA_{v}$. Q.E.D.

A projection $p$ is neutral if and only if $p^{\sharp} p\left(=p p^{\sharp}\right)=0$. Therefore if bounded operators $x$ and $x^{\#}$ leave the neutral subspace $p \Re$ invariant, then $x$ and the metric operator $J$ are represented in the form of $3 \times 3$ matrices of operators:

$$
\left(\begin{array}{lll}
x_{11} & x_{12} & x_{13} \\
0 & x_{22} & x_{23} \\
0 & 0 & x_{33}
\end{array}\right) \text { and }\left(\begin{array}{lll}
0 & 0 & J_{13} \\
0 & J_{22} & 0 \\
J_{31} & 0 & 0
\end{array}\right)
$$

where $p_{1}=p, p_{2}=1-p-p^{\sharp}, p_{3}=p^{\sharp}$ and where $x_{i j}$ and $J_{i j}$ are restrictions of $p_{i} x$ and $p_{i} J$ to $p_{j} \Re$, respectively. Furthermore $J_{22}$ is a selfadjoint unitary on $p_{2} \Re$, i.e. $\left\{p_{2} \Re, J_{22}\right\}$ is a Krein space.

If $x$ is unbounded and if the neutral subspaces $p \Re$ and $p^{\#} \Re$ are contained in the domain $D(x)$, then 


$$
D(x)=p \Re+\left\{D(x) \cap\left(1-p-p^{\#}\right) \Re\right\}+p^{\#} \Re
$$

and $x$ is represented in the form of a triangular matrix as shown in the above. More precise discussion on triangular matrices for unbounded operators will be done in Section 5 .

Definition 2.5 ([12], 15]). The above upper triangular matrix obtained from $x$ is called a canonical triangular matrix for $x$ if $x_{22}$ is \#-spectral or quasi-\#-spectral in a Krein space $\left\{p_{2} \Re, J_{22}\right\}$.

In $[10,11,12,13]$ the canonical triangular matrix is called a Tomita's triangular matrix.

Using these terminologies we can state our previous result, which will be used in Theorems 5.3 and 5.5.

THEOREM 2.6 ([12]). If $u$ is a bounded \#-unitary in a Krein space with $u-J u J$ compact, then it is represented by a canonical triangular matrix. In this case, $u_{22}$ is quasi-\#-spectral. In particular, if $\Re$ is a Pontrjagin space, then $u_{22}$ is \#-spectral.

\section{$\S 3$. Bounded \#-selfadjoint operators in a Krein sapce}

In this section we shall generalize the canonical triangular matrix representation for a bounded \#-selfadjoint operator in a Pontrjagin space to that in a Krein space.

THEOREM 3.1. If $h$ is a bounded \#-selfadjoint operator in a Krein space with $\mathrm{J}^{+} h J^{-}$compact, then it is represented by a canonical triangular matrix. In this case, $h_{22}$ is quasi-\#-spectral. In particular, if $\mathfrak{K}$ is a Pontrjagin space, then $h_{22}$ is \#-spectral.

The compactness of $J^{+} h J^{-}$is equivalent to that of $h-J h J$.

In $[10,16]$ we announced this statement without proof. The proof will proceed along the same line as it is done on a Pontrjagin space in [15]. However, a \#-unitary operator in a Krein space is not necessarily bounded. There gives rise to the following problems:

(i) How can one extend the concept of the \#-spectrality?

(ii) Since a \#-unitary operator $v$ which makes a given \#-selfadjoint operator $h \#$-spectral is not necessarily bounded, $v h v^{\#}$ may be unbounded in general even if $h$ is bounded. How can one find the selfadjoint extension?

The first one is already settled in Definition 2.3, whose appropriateness will be recognized through the discussion of our main theorems. The second one will be solved in Lemmas 3.4 and 3.5 below. 
For the proof of our theorem we must prepare the subsequent five lemmas.

LEMma 3.2 ([15]). If a Krein space is finite dimensional, then Theorem 3.1 holds.

The proof of this lemma was already given in [15] by showing that $\left(1-p-p^{\sharp}\right) h\left(1-p-p^{\sharp}\right)$ is diagonalizable for any maximal $h$-invariant neutral projection $p$. On the other hand, if we use the normal form of a \#-selfadjoint operator in a finite dimensional Krein space, we can also give another proof.

In this paper the operator inequality $x>0$ means that $x \geq 0$ and that $x \xi$ $=0$ implies $\xi=0$.

LEMMA 3.3. For a \#-selfadjoint operator $h$ in a Krein space, the following two conditions are equivalent:

(i) there exists a \#-unitary $v$ such that

a) $D\left(v^{*} v\right) \subset D(h)$ and $h D\left(v^{*} v\right) \subset D\left(v^{*} v\right)$,

b) $v h v^{*} \mid D_{0}$ is symmetric, where $D_{0}=v D\left(v^{*} v\right)$; and

(ii) there exists an operator $T \in \mathscr{L}(\Re)$ such that

c) $0<T<1$ and $J T J=1-T$,

d) $T h(1-T)$ is bounded and selfadjoint.

Proof. (i) $\rightarrow$ (ii): Let $v$ be a $\#$-unitary satisfying condition (i) and $k$ the restriction of $v h v^{\sharp}$ to $D_{0}$. Then $k$ is symmetric and

$$
v^{*} k v=v^{*} v h \quad \text { on } D\left(v^{*} v\right)
$$

by b) and a). If $\xi, \eta \in D\left(v^{*} v\right)$, then

$$
\begin{aligned}
\left(v^{*} k v \xi \mid \eta\right) & =(k v \xi \mid v \eta)=(v \xi \mid k v \eta)=\left(v \xi \mid v h v^{\sharp} v \eta\right) \\
& =(v \xi \mid v h \eta)=\left(v^{*} v \xi \mid h \eta\right)=\left(h^{*} v^{*} v \xi \mid \eta\right),
\end{aligned}
$$

where the last equality follows from a) and Remark c) for Definition 2.3. Thus

$$
v^{*} v h=v^{*} k v=h^{*} v^{*} v \quad \text { on } D\left(v^{*} v\right) .
$$

Now, we define an operator $T$ by

$$
T=v^{*} v\left(1+v^{*} v\right)^{-1} \text {. }
$$

Then $D(T)=\Re$ and $R(T)=R\left(v^{*} v\right)$. Since $1-T=\left(1+v^{*} v\right)^{-1}$, we have $D(1-T)$ $=\Omega$ and $R(1-T)=D\left(v^{*} v\right)$. As $v$ is $\#$-unitary, we see that $0<T<1$ and $J T J$ $=1-T$. Multiplying (3.1) by $\left(1+v^{*} v\right)^{-1}$ from the both sides, we have

$$
T h(1-T)=(1-T) h * T,
$$

both sides of which are defined everywhere on $\Re$ by a). Since $R\left(v^{*} v\right) \subset D\left(h^{*}\right)$, 
we have

$$
\begin{aligned}
(T h(1-T) \xi \mid \eta) & =\left(h(1-T) \xi \mid T_{\eta}\right)=\left((1-T) \xi \mid h^{*} T_{\eta}\right) \\
& =\left(\xi \mid(1-T) h^{*} T_{\eta}\right)=(\xi \mid T h(1-T) \eta)
\end{aligned}
$$

for all $\xi, \eta$ in $\Re$. It follows that the operator $T h(1-T)$ is selfadjoint. Hence it is bounded.

(ii) $\rightarrow$ (i): Let $T$ be the operator satisfying condition (ii). We set

$$
v=\left\{T(1-T)^{-1}\right\}^{1 / 2} .
$$

Then $v$ is invertible by c). Since $T h(1-T)$ is bounded by d), it follows that $D\left(v^{2}\right)=R(1-T) \subset D(h)$. Since

$$
J T h(1-T) J=(1-T) h * T
$$

by c) and $T h(1-T)$ is selfadjoint by d), it follows that $R\left(v^{2}\right)=R(T) \subset D\left(h^{*}\right)$ and $(1-T) h^{*} T$ is selfadjoint. Since

$$
\begin{aligned}
(T h(1-T) \xi \mid \eta) & =(\xi \mid T h(1-T) \eta)=(T \xi \mid h(1-T) \eta) \\
& =\left(h^{*} T \xi \mid(1-T) \eta\right)=\left((1-T) h^{*} T \xi \mid \eta\right)
\end{aligned}
$$

for all $\xi, \eta$ in $\Omega$, we can conclude that $T h(1-T)=(1-T) h^{*} T$. Therefore $(1-T)^{-1} T h(1-T)=h^{*} T$ and hence

$$
v^{2} h=h * v^{2} \quad \text { on } D\left(v^{2}\right),
$$

which implies $h D\left(v^{2}\right) \subset D\left(v^{2}\right)$. Thus a) is proved. Moreover, (3.2) gives us that $v^{2} h v^{*}=h^{*} v$ on $D_{0}$ and hence

$$
v h v^{\sharp}=v^{\sharp} h * v \quad \text { on } D_{0} .
$$

If $\xi, \eta$ are in $D_{0}$, then

$$
\left(v^{\sharp} h^{*} v \xi \mid \eta\right)=\left(h^{*} v \xi \mid v^{\sharp} \eta\right)=\left(v \xi \mid h v^{\sharp} \eta\right)=\left(\xi \mid v h v^{\sharp} \eta\right) .
$$

Hence $v h v^{\sharp}=\left(v h v^{\sharp}\right) *$ on $D_{0}$.

Q.E.D.

We will be able to show in Lemma 3.5 that, if $h$ is bounded, then $v h v^{\#} \mid D_{0}$ is essentially selfadjoint. For this we will prepare the following lemma.

Lemma 3.4. Let $a$ and $h$ be elements in $\mathscr{L}(\mathfrak{S})$ for some Hilbert space $\mathfrak{S}$. If $0<a<1$ and

$$
\left(a h a^{-1} \xi \mid \xi^{\prime}\right)=\left(\xi \mid a h a^{-1} \xi^{\prime}\right) \quad \text { for } \xi, \xi^{\prime} \in R(a),
$$

then

(i) $a h a^{-1}$ is essentially selfadjoint, 
(ii) $a^{-1} R\left(a^{2}\right)$ is a core of $a h a^{-1}$, and

(iii) $a h a^{-1} \subset a^{-1} h^{*} a$.

Proof. (i) Fix an arbitrary $\lambda \in i R$ with $|\lambda|>\|h\|$. Then $\pm \lambda \notin \mathrm{Sp}(h)$ and $h \pm \lambda 1$ has the bounded inverse. Thus $R(h \pm \lambda 1)=\mathscr{F}$. Since $D\left(a h a^{-1}\right)=R(a)$, it follows that

$$
R\left(a h a^{-1} \pm \lambda 1\right)=a(h \pm \lambda 1) a^{-1} R(a)=a(h \pm \lambda 1) \mathscr{S}=a \mathscr{S}=R(a) .
$$

Therefore the ranges of $a h a^{-1} \pm \lambda 1$ are dense in $\mathfrak{S}$. Thus $a h a^{-1}$ is essentially selfadjoint.

(ii) Let $h^{\prime}$ be the restriction of $a h a^{-1}$ to $a^{-1} R\left(a^{2}\right)$. Then

$$
R\left(h^{\prime} \pm \lambda 1\right)=a(h \pm \lambda 1) a^{-1} a^{-1} R\left(a^{2}\right)=a(h \pm \lambda 1) \mathfrak{S}=R(a),
$$

which is dense in $\mathfrak{S}$. Thus $h^{\prime}$ is also essentially selfadjoint. Since $h^{\prime} \subset a h a^{-1}$, both $a h a^{-1}$ and $h^{\prime}$ have the same closure. Thus $a^{-1} R\left(a^{2}\right)$ is a core of $a h a^{-1}$.

(iii) Rewrite the right hand side of (3.3) in the form $\left(h^{*} a \xi \mid a^{-1} \xi^{\prime}\right)$. Since this is continuous in $\xi^{\prime}$ by the left hand side of (3.3), it follows that $h^{*} a \xi \epsilon$ $D\left(a^{-1}\right)$ and $a h a^{-1} \xi=a^{-1} h^{*} a \xi$ for $\xi \in R(a)$.

Q.E.D.

LEMMA 3.5. Assume condition (i) in Lemma 3.3 for a \#-selfadjoint operator $h$ and $a \#$-unitary $v$. If $h$ is bounded, then $v h v^{\#} \mid D_{0}$ is essentially selfadjoint.

Proof. We may assume that $v$ is positive and selfadjoint by polar decomposition. Let $p_{1}, p_{2}$ and $p_{3}$ be the spectral projections of $v$ corresponding to the intervals $(0,1),\{1\}$ and $(1, \infty)$, respectively. Since $J v J=v^{-1}$, we have $J p_{1} J=p_{3}$ and $J p_{2} J=p_{2}$. Here we set

$$
a=p_{1} v\left|p_{1} \Re, \quad J_{i j}=p_{i} J\right| p_{j} \Re, \quad h_{i j}=p_{i} h \mid p_{j} \Re .
$$

Utilizing these operators, we represent $v$ and $h$ in the form of matrices

$$
v=\left(\begin{array}{ccc}
a & 0 & 0 \\
0 & 1 & 0 \\
0 & 0 & J_{31} a^{-1} J_{13}
\end{array}\right), \quad h=\left(h_{i j}\right)
$$

on $\AA$. Since $h$ is \#-selfadjoint, it follows that $J_{31} h_{11}^{*} J_{13}=h_{33}$.

Let $k$ be the restriction of $v h v^{-1}$ to $D_{0}=v D\left(v^{*} v\right)$. Then we see that

$$
k=\left(\begin{array}{ccc}
a h_{11} a^{-1} & a h_{12} & a h_{13} J_{31} a J_{13} \\
h_{21} a^{-1} & h_{22} & h_{23} J_{31} a J_{13} \\
J_{31} a^{-1} J_{13} h_{31} a^{-1} & J_{31} a^{-1} J_{13} h_{32} & J_{31} a^{-1} J_{13} h_{33} J_{31} a J_{13}
\end{array}\right)
$$

on $D_{0}$ and 


$$
\begin{aligned}
D_{0} & =v D\left(v^{*} v\right)=v\left\{\left(p_{1}+p_{2}\right) \Re+J_{31} R\left(a^{2}\right)\right\} \\
& =R(a)+p_{2} \Re+J_{31} a^{-1} R\left(a^{2}\right) .
\end{aligned}
$$

Since $k$ is symmetric by assumption, we obtain

$$
\left(k\left(\xi_{1}+\xi_{2}+\xi_{3}\right) \mid \xi_{1}^{\prime}+\xi_{2}^{\prime}+\xi_{3}^{\prime}\right)=\left(\xi_{1}+\xi_{2}+\xi_{3} \mid k\left(\xi_{1}^{\prime}+\xi_{2}^{\prime}+\xi_{3}^{\prime}\right)\right)
$$

for any $\xi_{1}, \xi_{1}^{\prime} \in R(a), \xi_{2}, \xi_{2}^{\prime} \in p_{2} \Re$ and $\xi_{3}, \xi_{3}^{\prime} \in J_{31} a^{-1} R\left(a^{2}\right)$. Therefore we have

$$
\begin{aligned}
\left(a h_{11} a^{-1} \xi_{1} \mid \xi_{1}^{\prime}\right) & =\left(\xi_{1} \mid a h_{11} a^{-1} \xi_{1}^{\prime}\right) \\
\left(a h_{12} \xi_{2} \mid \xi_{1}^{\prime}\right) & =\left(\xi_{2} \mid h_{21} a^{-1} \xi_{1}^{\prime}\right) \\
\left(a h_{13} J_{31} a J_{13} \xi_{3} \mid \xi^{\prime}\right) & =\left(\xi_{3} \mid J_{31} a^{-1} J_{13} h_{31} a^{-1} \xi_{1}^{\prime}\right) \\
\left(h_{23} J_{31} a J_{13} \xi_{3} \mid \xi_{2}^{\prime}\right) & =\left(\xi_{3} \mid J_{31} a^{-1} J_{13} h_{32} \xi_{2}^{\prime}\right)
\end{aligned}
$$

for any $\xi_{1}, \xi_{1}^{\prime} \in R(a), \xi_{2}, \xi_{2}^{\prime} \in p_{2} \Re$ and $\xi_{3} \in J_{31} a^{-1} R\left(a^{2}\right)$. According to Lemma 3.4 $a h_{11} a^{-1}$ is essentially selfadjoint on $p_{1} \Re$ and $a h_{11} a^{-1} \subset a^{-1} h_{11}^{*} a$. Moreover, we have

$$
\begin{gathered}
\left\|h_{21} a^{-1} \xi_{1}^{\prime}\right\| \leq\left\|a h_{12}\right\|\left\|\xi_{1}^{\prime}\right\| \\
\left\|J_{31} a^{-1} J_{13} h_{31} a^{-1} \xi_{1}^{\prime}\right\| \leq\left\|a h_{13} J_{31} a J_{13}\right\|\left\|\xi_{1}^{\prime}\right\| \\
\left\|J_{31} a^{-1} J_{13} h_{32} \xi_{2}^{\prime}\right\| \leq\left\|h_{23} J_{31} a J_{13}\right\|\left\|\xi_{2}^{\prime}\right\|
\end{gathered}
$$

for any $\xi_{1}^{\prime} \in R(a)$ and $\xi_{2}^{\prime} \in p_{2} \Re$, and hence

$$
\left\{\begin{aligned}
\left(h_{21} a^{-1}\right)^{-} & =\left(a h_{12}\right)^{*} \\
\left(J_{31} a^{-1} J_{13} h_{31} a^{-1}\right)^{-} & =\left(a h_{13} J_{31} a J_{13}\right)^{*} \\
\left(J_{31} a^{-1} J_{13} h_{32}\right)^{-} & =\left(h_{23} J_{31} a J_{13}\right)^{*},
\end{aligned}\right.
$$

where $b^{-}$denotes the closure of $b$. Here we set

$$
k_{0}=\left(\begin{array}{ccc}
\left(a h_{11} a^{-1}\right)^{-} & 0 & 0 \\
0 & h_{22} & 0 \\
0 & 0 & J_{31}\left(a h_{11} a^{-1}\right)^{-} J_{13}
\end{array}\right)
$$

and

$$
k_{1}=\left(\begin{array}{ccc}
0 & a h_{12} & a h_{13} J_{31} a J_{13} \\
\left(a h_{12}\right)^{*} & 0 & h_{23} J_{31} a J_{13} \\
\left(a h_{13} J_{31} a J_{13}\right)^{*} & \left(h_{23} J_{31} a J_{13}\right)^{*} & 0
\end{array}\right) .
$$

The diagonal part of $k \mid D_{0}$ is different from that of $k_{0}$ at the $(3,3)$ component. The off diagonal part of $k \mid D_{0}$ is different from that of $k_{1}$ at the lower triangular part. We compare the $(3,3)$ components of $k \mid D_{0}$ and $k_{0}$. Since $a^{-1} h_{11}^{*} a\left|a^{-1} R\left(a^{2}\right)=a h_{11} a^{-1}\right| a^{-1} R\left(a^{2}\right) \subset a h_{11} a^{-1}$, it follows from (3.4) that $k \subset k_{0}+k_{1}$. 
Since $k_{0}$ is selfadjoint and $k_{1}$ is bounded selfadjoint by (3.4), the Kato-Rellich theorem [6] tells us that $k_{0}+k_{1}$ is essentially selfadjoint. Since $a^{-1} R\left(a^{2}\right)$ is a core of $a h_{11} a^{-1}$ by Lemma 4.3, it follows that $D_{0}$ is a core of $k_{0}$ and hence of $k_{0}+k_{1}$. Thus $k^{-}=\left(k_{0}+k_{1}\right)^{-}$is selfadjoint.

Q.E.D.

From this lemma we know that if a bounded \#-selfadjoint operator $h$ satisfies condition (ii) in Lemma 3.3, then $h$ is quasi-\#-spectral.

REMARK. Let $\{\Re,\langle\rangle$,$\} be a Krein space. Let h$ be a bounded \#-selfadjoint operator and $p$ a maximal $h$-invariant neutral projection. If $h^{\prime}$ and $J^{\prime}$ are restrictions of $h$ and $J$ to the subspace $\left(1-p-p^{\#)} \Re\right.$, and if $h^{\prime}-J^{\prime} h^{\prime} J^{\prime}$ is compact, then $h^{\prime}$ is quasi-\#-spectral.

LemMA 3.6. For a bounded \#-selfadjoint operator $h$ the following two conditions are equivalent:

(i) $h$ is represented by a canonical triangular matrix; and

(ii) there exists an operator $T$ such that

a) $0 \leq T \leq 1$ and $J T J=1-T$,

b) $T h(1-T)$ is selfadjoint.

The proof of this lemma is completed by the same argument done for a Pontrjagin space [15] by representing $T$ and $h$ in the forms of $3 \times 3$ matrices by means of spectral projections of $T$ corresponding to the intervals $\{0\},(0,1)$ and $\{1\}$, respectively.

Proof of Theorem 3.1. Let $h$ be a bounded \#-selfadjoint operator with $h$-JhJ compact. Then $h$ is of the form

$$
\left(\begin{array}{ll}
h_{11} & h_{12} \\
h_{21} & h_{22}
\end{array}\right),
$$

where $p_{1}=J^{+}, p_{2}=J^{-}$and $h_{i j}$ is the restriction of $p_{i} h$ to $p_{j} \AA$. A pair

$$
\left\{\left(\begin{array}{cc}
h_{11} & 0 \\
0 & h_{22}
\end{array}\right), \quad h_{21}\right\}
$$

of a bounded selfadjoint operator on $\Re$ and a compact operator of $\Re^{+}$to $\AA^{-}$ is an element in the topological product space

$$
\left\{\mathscr{L}\left(\Re^{+}\right) \oplus \mathscr{L}\left(\Re^{-}\right) \text {, *-strong top. }\right\} \times\left\{\mathscr{L} \mathscr{C}\left(\Re^{+}, \Re^{-}\right) \text {, norm top. }\right\}
$$

Since the set of operators of finite rank is dense in the above set, we can approximate the above pair by a net of pairs 


$$
\left\{\left(\begin{array}{cc}
h_{11}^{(i)} & 0 \\
0 & h_{22}^{(i)}
\end{array}\right), h_{21}^{(i)}\right\}, \quad i \in I
$$

of a finite rank selfadjoint operator and a finite rank operator such that

$$
\left\|\left(\begin{array}{cc}
h_{11}^{(i)} & 0 \\
0 & h_{22}^{(i)}
\end{array}\right)\right\| \leq\left\|\left(\begin{array}{cc}
h_{11} & 0 \\
0 & h_{22}
\end{array}\right)\right\| .
$$

The last inequality is assured by the Kaplansky's density theorem. The operator $h_{i}$ defined by

$$
\left(\begin{array}{cc}
h_{11}^{(i)} & -h_{21}^{(i)} * \\
h_{21}^{(i)} & h_{22}^{(i)}
\end{array}\right), \quad i \in I
$$

is a \#-sel'adjoint operator of finite rank. There exists a \#-projection $e_{i}$ of finite rank such that $h_{i}=h_{i} e_{i}$. Since $\left\{e_{i} \Omega,\langle,\rangle_{e_{i} \Omega}\right\}$ is a Krein space, the restriction $h_{i}^{\prime}$ of $h_{i}$ to $e_{i} \Re$ is represented by a canonical triangular matrix by Lemma 3.2. Hence $h_{i}$ itself is represented by a canonical triangular matrix by extending the $(2,2)$ component of a canonical triangular matrix of $h_{i}^{\prime}$ by defining it to be 0 on $\left(1-e_{i}\right) \Re$. Then there exists for each $h_{i}$ an operator $T_{i}$ which satisfies conditions a) and b) in Lemma 3.6. By means of a) the $\operatorname{set}\left\{T_{i}: i \in I\right\}$ is relatively $\sigma\left(\mathscr{L}(\Re), \mathscr{L}(\Re)_{*}\right)$-compact. It contains a convergent net $\left\{T_{j}: j \in I^{\prime}\right\}$ in $\sigma\left(\mathscr{L}(\Re), \mathscr{L}(\Re)_{*}\right)$-topology. Clearly, the limit $T$ satisfies condition a). According to Lemma 3.6 it remains to show that $T$ also satisfies condition $b$ ).

Now, we rewrite the above net by $\left\{T_{i}: i \in I\right\}$. Then condition b) for $T_{i}$ is equivalent to

$$
T_{i} h_{i}-h_{i}^{*} T_{i}=T_{i}\left(h_{i}-h_{i}^{*}\right) T_{i}
$$

The left hand side converges $T h-h^{*} T$ in $\sigma\left(\mathscr{L}(\Re), \mathscr{L}(\Re)_{*}\right)$-topology. Indeed, $T_{i} h_{i}-T h=T_{i}\left(h_{i}-h\right)+\left(T_{i}-T\right) h$. Thus our problem is reduced to show that the right hand side converges to $T\left(h-h^{*}\right) T$ in $\sigma\left(\mathscr{L}(\Re), \mathscr{L}(\Re)_{*}\right)$-topology.

Since $h-h^{*}=h-J h J$ is compact by assumption, for any $\varepsilon>0$ there exists an operator of finite rank

$$
k_{0}=\left(\begin{array}{cc}
0 & -k_{21}^{*} \\
k_{21} & 0
\end{array}\right)
$$

with $\left\|(h-J h J)-k_{0}\right\|<\varepsilon / 8$. For the notational convenience we write the off diagonal component of $h_{i}$ and $h$ by

$$
k_{i}=h_{i}-J h_{i} J, \quad k=h-J h J .
$$

Then 


$$
\begin{aligned}
T_{i} k_{i} T_{i}-T k T=T_{i}\left(k_{i}-k\right) T_{i} & +\left(T_{i}-T\right)\left(k-k_{0}\right) T_{i} \\
& +\left(T_{i}-T\right) k_{0} T_{i}+T k\left(T_{i}-T\right) .
\end{aligned}
$$

According to our choice of the net, $k_{i}$ converges to $k$ in norm and $T_{i}$ converges to $T$ in $\sigma\left(\mathscr{L}(\Re), \mathscr{L}(\Re)_{*}\right)$-topology. Since $k_{0}$ is of finite rank, $\left(T_{i}-T\right) k_{0} T_{i}$ converges to zero in norm. Thus for any $\varepsilon>0$ in the above and for any unit vectors $\xi, \eta$ in $\Re$, there exists an $i_{0} \in I$ such that if $i>i_{0}$ then

$$
\left\|k_{i}-k\right\|<\varepsilon / 4, \quad\left|\left(\left(T_{i}-T\right) k_{0} T_{i} \xi \mid \eta\right)\right|<\varepsilon / 4
$$

and

$$
\left|\left(\left(T_{i}-T\right) \xi \mid k^{*} T_{\eta}\right)\right|<\varepsilon / 4 .
$$

Consequently, for $i>i_{0}$, we have

$$
\left|\left(\left(T_{i} k_{i} T_{i}-T k T\right) \xi \mid \eta\right)\right|<\varepsilon,
$$

which implies that $T_{i} k_{i} T_{i}$ converges to $T k T$ in $\sigma\left(\mathscr{L}(\Re), \mathscr{L}(\Re)_{*}\right)$-topology.

Q.E.D.

\section{§4. Cayley transform in a Krein space}

First we shall recall the Cayley transform in a Krein space, [4]. Let $\lambda$ be a nonreal complex number. If $h$ is a $\#$-selfadjoint operator and $\lambda \notin \mathrm{Sp}(h)$, then the Cayley transform

$$
u=(h-\bar{\lambda} 1)(h-\lambda 1)^{-1}
$$

is a bounded \#-unitary and 1 is not in the point spectrum of $u$. Conversely, if $u$ is a bounded \#-unitary and 1 is not in the point spectrum, then the Cayley transform

$$
h=(\lambda u-\bar{\lambda} 1)(u-1)^{-1}
$$

is \#-selfadjoint and $\lambda \notin \mathrm{Sp}(h)$. In addition, the Cayley transform of the Cayley transform of a \#-selfadjoint operator $h$ coincides with $h$ itself. The same is true for a bounded \#-unitary.

REMARK. We shall give an example of a \#-selfadjoint operator whose spectrum is the whole complex plane. Thus we cannot consider the Cayley transform for such an operator.

Let $\left\{\lambda_{i} ; i \in N\right\}$ be a conutable dense subset of $\boldsymbol{C} \backslash \boldsymbol{R}$. The direct sum

$$
h=\sum_{i \in N}^{\oplus}\left(\begin{array}{ll}
\lambda_{i} & 0 \\
0 & \bar{\lambda}_{i}
\end{array}\right)
$$


in a Krein space

$$
\sum_{i \in \boldsymbol{N}} \oplus\left\{\mathscr{\mathcal { C }}_{i}, J_{i}\right\} \quad\left(\operatorname{dim} \tilde{S}_{i}=2, J_{i}=\left(\begin{array}{ll}
0 & 1 \\
1 & 0
\end{array}\right)\right)
$$

is a $\#$-selfadjoint operator with $\operatorname{Sp}(h)=C$.

Before going into our main theorem we shall discuss the domains of unbounded operators.

If the domain $D$ of an operator $a$ satisfies

$$
D=D \cap \Re^{+}+D \cap \Re^{-},
$$

then $a$ is represented by a $2 \times 2$ matrix of operators:

$$
\left(\begin{array}{ll}
a_{11} & a_{12} \\
a_{21} & a_{22}
\end{array}\right),
$$

where $p_{1}=J^{+}, p_{2}=J^{-}$and $a_{i j}=p_{i} a \mid p_{j} \Re$. Moreover, $J D=D$. $D(a)$.

An operator $a$ is said to be \#-symmetric if $\langle a \xi, \eta\rangle=\langle\xi, a \eta\rangle$ for all $\xi, \eta \in$

Lemma 4.1. Let a be a closed \#-symmetric operator with dense domain $D$. If $D$ satisfies (4.1) and $a$ is represented in the form (4.2), then

(i ) $a_{11}$ and $a_{22}$ are densely defined symmetric operators in $\AA^{+}$and $\AA^{-}$ respectively,

(ii) $a_{21} \subset-a_{12}^{*}$ and $a_{12} \subset-a_{21}^{*}$; and

(iii) if $a_{21}$ (resp. $\left.a_{12}, a_{11}, a_{22}\right)$ is bounded, then $a_{11}$ (resp. $\left.a_{22}, a_{21}, a_{12}\right)$ is crosed.

Proof. (i ) Since $p_{1} D=D \cap \Re^{+}$by (4.1), we have for any $\xi, \eta \in D \cap \Re^{+}$

$$
\begin{aligned}
\left(a_{11} \xi \mid \eta\right)_{\Re+} & =(a \xi \mid \eta)=\langle a \xi, \eta\rangle=\langle\xi, a \eta\rangle \\
& =(\xi \mid a \eta)=\left(\xi \mid a_{11} \eta\right)_{\Re+} .
\end{aligned}
$$

Therefore $a_{11}$ is symmetric. By symmetry, $a_{22}$ is also symmetric.

(ii) For any $\xi \in D \cap \Re^{+}$and $\eta \in D \cap \Re^{-}$we have

$$
-\left(a_{21} \xi \mid \eta\right)=\langle a \xi, \eta\rangle=\langle\xi, a \eta\rangle=\left(\xi \mid a_{12} \eta\right) .
$$

Thus $a_{12} \subset-a_{21}^{*}$ and $a_{21} \subset-a_{12}^{*}$.

(iii) Suppose that $\xi_{n} \in D \cap \mathfrak{R}^{+}, \xi_{n} \rightarrow \xi$ and $a_{11} \xi_{n} \rightarrow \eta$. If $a_{21}$ is bounded, then $a \xi_{n}=a_{11} \xi_{n}+a_{21} \xi_{n} \rightarrow \eta+\bar{a}_{21} \xi$, where $\bar{a}_{21}$ is the closure of $a_{21}$. Since $a$ is a closed operator, $\xi \in D$ and $a \xi=\eta+\bar{a}_{21} \xi$. Therefore $a_{11} \xi=\eta$ and hence $a_{11}$ is closed.

For the remaining operators the similar argument holds.

Q.E.D.

COROLlaRY 4.2. Let a be a closed \#-symmetric operator with dense domain 
$D$. If $D$ satisfies (4.1) and if $a_{12}$ and $a_{21}$ are bounded, then the following two coditions are equivalent:

(i) $a$ is \#-selfadjoint; and

(ii) $a_{11}$ and $a_{22}$ are selfadjoint in $\Re^{+}$and $\Re^{-}$respectively.

Proof. By means of Lemma 4.1, $a_{12}^{*}=-\bar{a}_{21}$ and $a_{21}^{*}=-\bar{a}_{12}$.

(i) $\rightarrow$ (ii): Suppose that $\eta \in D\left(a_{11}^{*}\right)$. If $\xi \in D$, then $\xi=\xi^{+}+\xi^{-}$for some $\xi^{ \pm}$ $\in D \cap \Re^{ \pm}$. Therefore

$$
\begin{aligned}
(a \xi \mid \eta) & =\left(a_{11} \xi^{+} \mid \eta\right)+\left(a_{12} \xi^{-} \mid \eta\right) \\
& =\left(\xi^{+} \mid a_{11}^{*} \eta\right)-\left(\xi^{-} \mid \bar{a}_{21} \eta\right) \\
& =\left(\xi \mid a_{11}^{*} \eta-\bar{a}_{21} \eta\right)
\end{aligned}
$$

and hence $\eta \in D\left(a^{*}\right)=J D=D$. Thus $\eta \in D \cap \Re^{+}=D\left(a_{11}\right)$ and hence $a_{11}^{*}=a_{11}$. Similarly, we can verify $D\left(a_{22}^{*}\right)=D\left(a_{22}\right)$ and hence $a_{22}^{*}=a_{22}$.

(ii) $\rightarrow$ (i): Suppose that $\eta \in D\left(a^{*}\right)$ and $\xi \in D$. Since $\xi$ and $\eta$ are of the forms $\xi=\xi^{+}+\xi^{-}$and $\eta=\eta^{+}+\eta^{-}$for some $\xi^{ \pm} \in D \cap \Re^{ \pm}$and $\eta^{ \pm} \in \Re^{ \pm}$, we have

$$
\begin{aligned}
\left(a_{11} \xi^{+} \mid \eta^{+}\right) & =\left(a_{11} \xi^{+} \mid \eta\right)=\left(a \xi^{+} \mid \eta\right)-\left(\bar{a}_{21} \xi^{+} \mid \eta\right) \\
& =\left(\xi^{+} \mid a^{*} \eta\right)+\left(\xi^{+} \mid \bar{a}_{12} \eta\right)=\left(\xi^{+} \mid a^{*} \eta+\bar{a}_{12} \eta\right) .
\end{aligned}
$$

Thus $\eta^{+} \in D\left(a_{11}^{*}\right)=D\left(a_{11}\right)$. Repeating the similar argument, we have $\eta^{-} \epsilon$ $D\left(a_{22}\right)$. Consequently we obtain

$$
\eta=\eta^{+}+\eta^{-} \in D \cap \Re^{+}+D \cap \Re^{-}=D,
$$

and hence $D\left(a^{\sharp}\right)=J D\left(a^{*}\right) \subset J D=D$. Thus $a=a^{\sharp}$.

Q.E.D.

Corollary 4.3. Let a be a closed \#-symmetric operator with dense domain $D$. If $\Re^{+} \subset D$, then

(i) $D=\Re^{+}+D \cap \Re^{-}$,

(ii) $a_{11}$ is selfadjoint and $a_{22}$ is symmetric, and

(iii) $a_{11}, a_{12}$ and $a_{21}$ are bounded.

In particular, $a$ is \#-selfadjoint if and only if $a_{22}$ is selfadjoint in $\Re^{-}$.

Proof. (i) and the fact that $a_{11}$ and $a_{22}$ are symmetric are clear. Since $a_{11}$ is closed and $D\left(a_{11}\right)=\Re^{+}$, it follows that $a_{11}$ is bounded. Therefore $a_{21}$ is closed by (iii) in Lemma 4.1 and hence it is bounded. Since $a_{12} \subset-a_{21}^{*}$ by (ii) in Lemma 4.1, $a_{12}$ is bounded.

The last part follows from Corollary 4.2.

Q.E.D.

In the following theorem we shall apply Corollary 4.3 to a \#-selfadjoint operator $h$ and the matrix representation $\left(h_{i j}\right)$ corresponding to (4.2).

TheOREM 4.4. Let $h$ be a \#-selfadjoint operator such that $\Re^{+} \subset D(h)$ and 
$\bar{h}_{12}\left(h_{22}-\lambda_{0} 1\right)^{-1}$ is compact for some $\lambda_{0} \notin \operatorname{Sp}\left(h_{22}\right)$. If $\lambda \in C$ satisfies $\left\|h_{12}\right\|<|\operatorname{Im} \lambda|$ and $\left\|h_{21}\right\|<|\operatorname{Im} \lambda|$, then $\lambda \notin \operatorname{Sp}(h) \cup \boldsymbol{R}$ and the Cayley transform $u=(h-\bar{\lambda} 1) \times$ $(h-\lambda 1)^{-1}$ is a bounded \#-unitary with $u-J u J$ compact.

Proof. Since $\mathfrak{R}^{+} \subset D(h)$, it follows from Corollary 4.3 that $h_{11}, \bar{h}_{12}$ and $h_{21}$ are bounded, $h_{11}$ and $h_{22}$ are selfadjoint, and $h_{12}^{*}=-h_{21}$. Since $|\operatorname{Im} \lambda|>0$, we have $\lambda \neq \bar{\lambda}$. Thus $h_{11}-\lambda 1$ and $h_{22}-\lambda 1$ are invertible. However

$$
\left\|\left(h_{11}-\lambda 1\right) \xi\right\|^{2}=\left\|\left\{h_{11}-(\operatorname{Re} \lambda) 1\right\} \xi\right\|^{2}+|\operatorname{Im} \lambda|^{2}\|\xi\|^{2}, \quad \xi \in \Re^{+}
$$

and

$$
\left\|\left(h_{22}-\lambda 1\right) \eta\right\|^{2}=\left\|\left\{h_{22}-(\operatorname{Re} \lambda) 1\right\} \eta\right\|^{2}+|\operatorname{Im} \lambda|^{2}\|\eta\|^{2}, \quad \eta \in D(h) \cap \Re^{-} .
$$

Thus we obtain

$$
\left\|\left(h_{j j}-\lambda 1\right)^{-1}\right\| \leq|\operatorname{Im} \lambda|^{-1} \quad \text { for } j=1,2 .
$$

We set

$$
\begin{aligned}
& b_{12}=\left(h_{11}-\lambda 1\right)^{-1} \bar{h}_{12} \quad \text { and } \quad b_{21}=\left(h_{22}-\lambda 1\right)^{-1} h_{21} \\
& c_{12}=\bar{h}_{12}\left(h_{22}-\lambda 1\right)^{-1} \quad \text { and } \quad c_{21}=h_{21}\left(h_{11}-\lambda 1\right)^{-1} .
\end{aligned}
$$

Then $b_{12}, c_{12}$ belong to $\mathscr{L}\left(\Re^{-}, \Re^{+}\right)$and $b_{21}, c_{21}$ belong to $\mathscr{L}\left(\Re^{+}, \Re^{-}\right)$. Since $\left\|h_{12}\right\|$ $<|\operatorname{Im} \lambda|$ and $\| h_{21}|<| \operatorname{Im} \lambda \mid$ by assumption, it follows that $\left\|b_{12} b_{21}\right\|<1,\left\|b_{21} b_{12}\right\|<1$, $\left\|c_{12} c_{21}\right\|<1$ and $\left\|c_{21} c_{12}\right\|<1$. Therefore $1-b_{12} b_{21}, 1-b_{21} b_{12}, 1-c_{12} c_{21}$ and $1-c_{21} c_{12}$ have bounded inverses. Here we define bounded operators $v_{1}$ and $v_{2}$ by

$$
\left(\begin{array}{cc}
\left(1-b_{12} b_{21}\right)^{-1} & 0 \\
0 & \left(1-b_{21} b_{12}\right)^{-1}
\end{array}\right)\left(\begin{array}{cc}
1 & -b_{12} \\
-b_{21} & 1
\end{array}\right)\left(\begin{array}{cc}
\left(h_{11}-\lambda 1\right)^{-1} & 0 \\
0 & \left(h_{22}-\lambda 1\right)^{-1}
\end{array}\right)
$$

and

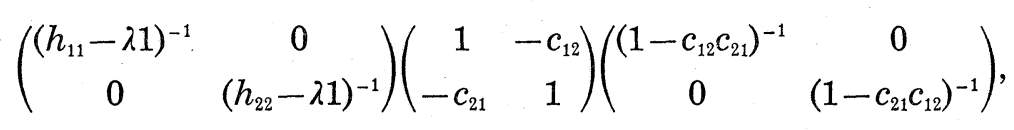

respectively. Then it is easy to see that $v_{1}(h-\lambda 1)=1$ on $D(h)$ and $(h-\lambda) v_{2}=1$. Thus $h-\lambda 1$ has the bounded inverse $v_{1}=v_{2}$ and so $\lambda \notin \operatorname{Sp}(h) \cup \boldsymbol{R}$.

The Cayley transform is expressed in the form

$$
u=(h-\bar{\lambda} 1)(h-\lambda 1)=1+(\lambda-\bar{\lambda})(h-\lambda 1)^{-1} .
$$

Therefore

$$
\begin{aligned}
u_{12} & =-(\lambda-\bar{\lambda})\left(1-b_{12} b_{21}\right)^{-1} b_{12}\left(h_{22}-\lambda 1\right)^{-1} \\
& =-(\lambda-\bar{\lambda})\left(1-b_{12} b_{21}\right)^{-1}\left(h_{11}-\lambda 1\right)^{-1} \bar{h}_{12}\left(h_{22}-\lambda 1\right)^{-1} .
\end{aligned}
$$


Let $R(\mu)$ denote the resolvent $\left(h_{22}-\mu 1\right)^{-1}$ of $h_{22}$ for $\mu \notin \operatorname{Sp}\left(h_{22}\right)$. Then $\bar{h}_{12} R\left(\lambda_{0}\right)$ is compact by assumption. Multiplying the resolvent equation $R\left(\lambda_{0}\right)-R(\lambda)=$ $\left(\lambda_{0}-\lambda\right) R\left(\lambda_{0}\right) R(\lambda)$ by $\bar{h}_{12}$ from the left, we find that $\bar{h}_{12} R(\lambda)$ is also compact. Hence $u_{12}$ is compact. Since $u$ is a bounded \#-unitary, $u_{21}=-u_{12}^{*}$. Consequently, $u-J u J$ is compact.

Q.E.D.

This theorem will be used in Theorem 5.5.

\section{§ 5. Unbounded \#-selfadjoint operators}

Let $a$ be a closed operator with dense domain $D$. For projections $p_{1}, p_{2}$ and $p_{3}$ with $p_{1}+p_{2}+p_{3}=1$ we denote

$$
\left\{\begin{array}{l}
\Re_{i}=p_{i} \Re \quad(i=1,2,3) \\
D_{0}=D \cap \Re_{1}+D \cap \Re_{2}+D \cap \Re_{3} .
\end{array}\right.
$$

Then $D_{0}(\subset D)$ may happen to be $\{0\}$. Let $a_{0}$ be the matrix $\left(a_{i j}\right)$ of operators obtained by

$$
a_{i j}=p_{i} a \mid D \cap \Re_{j} \quad(i, j=1,2,3) .
$$

Since the domain of the matrix is $D_{0}$, if $D=D_{0}$, then $a=a_{0}$, However, if $D_{0}$ is not a core of $a$, then it will be difficult to reconstruct $a$ from $a_{0}$ and the matrix representation $a_{0}$ has no meaning for the' given operator $a$. For this reason we shall restrict ourselves to the case where $D_{0}$ is a core of $a$.

Now, suppose that a bounded operator $v$ has a densely defined inverse $v^{-1}$. The inverse of the closed operator is closed in general. We shall apply the above discussion to $v^{-1}$. Let $D$ denote $D\left(v^{-1}\right)=R(v)$. If the matrix representation of $v$ is of the form

$$
\left(\begin{array}{lll}
v_{11} & v_{12} & v_{13} \\
0 & v_{22} & v_{23} \\
0 & 0 & v_{33}
\end{array}\right)
$$

then

$$
\left\{\begin{array}{l}
D \cap \Re_{1}=R\left(v_{11}\right)+\left\{v_{12} \xi+v_{13} \eta: \xi \in \Re_{2}, \eta \in \operatorname{Ker} v_{33}, v_{22} \xi+v_{23} \eta=0\right\} \\
D \cap \Re_{2}=\left\{v_{22} \xi+v_{23} \eta: \xi \in \Re_{2}, \eta \in \operatorname{Ker} v_{33}, v_{12} \xi+v_{13} \eta \in R\left(v_{11}\right)\right\} \\
D \cap \Re_{3}=v_{33}\left\{\eta \in \Re_{3}: v_{12} \xi+v_{13} \eta \in R\left(v_{11}\right), v_{22} \xi+v_{23} \eta=0 \text { for some } \xi \in \Re_{2}\right\}
\end{array}\right.
$$

and

$$
\left\{\begin{array}{l}
p_{1} D=R\left(v_{11}\right)+R\left(v_{12}\right)+R\left(v_{13}\right) \\
p_{2} D=R\left(v_{22}\right)+R\left(v_{23}\right) \\
p_{3} D=R\left(v_{33}\right) .
\end{array}\right.
$$


Since $D$ is dense in $\Re, R\left(v_{33}\right)$ is dense in $\AA_{3}$ by (5.5). Since $v$ is invertible, $\operatorname{Ker} v_{11}=\{0\}$. Moreover, if $\operatorname{dim} \Re_{1}<\infty$ and $\operatorname{dim} \Re_{3}<\infty$, then $R\left(v_{11}\right)=\Re_{1}$ and $R\left(v_{33}\right)=\Omega_{3}$. Hence $D \cap \Omega_{1}=\Re_{1}$ by (5.4) and Ker $v_{33}=\{0\}$.

LEMMA 5.1. Let $v$ be a bounded operator with densely defined inverse. If $\left(v_{i j}\right)$ is the matrix representation (5.3) of $v$ and if

$$
D_{0}=D \cap \Re_{1}+D \cap \Re_{2}+D \cap \Re_{3} \quad\left(D=D\left(v^{-1}\right)\right)
$$

is a core of $v^{-1}$ and $\left(c_{i j}\right)$ the matrix representation of $v^{-1}$, then the following two conditions are equivalent:

(i) $\operatorname{Ker} v_{33}=\{0\}$; and

(ii) $c_{31}=0, c_{32}=0$ and $c_{33}$ is closable.

In this case, $v_{33}$ has the densely defined inverse and $\bar{c}_{33}=v_{33}^{-1}$.

Proof. (i) $\rightarrow$ (ii): Suppose that $\operatorname{Ker} v_{33}=\{0\}$. Then the inverse $v_{33}^{-1}$ exists. Using the matrix representation $\left(c_{i j}\right)$ of $v^{-1}$, we rewrite the equality $v v^{-1}=1$ on $D_{0}$ in the matrix form. Then we have

$$
\left(\begin{array}{ccc}
v_{11} c_{11}+v_{12} c_{21}+v_{13} c_{31} & v_{11} c_{12}+v_{12} c_{22}+v_{13} c_{32} & v_{11} c_{13}+v_{12} c_{23}+v_{13} c_{33} \\
v_{22} c_{21}+v_{23} c_{31} & v_{22} c_{22}+v_{23} c_{32} & v_{22} c_{23}+v_{23} c_{33} \\
v_{33} c_{31} & v_{33} c_{32} & v_{33} c_{33}
\end{array}\right)=1
$$

on $D_{0}$. Since $v_{33}$ is injective, we get $c_{3 i}=0$ on $D \cap \Re_{i}$ for $i=1,2$. Furthermore

$$
v_{33} c_{33}=1 \quad \text { on } D \cap \AA_{3} .
$$

If $\xi \in D \cap \Re_{3}$ and $\eta \in \Re_{3}$, then

$$
\left(c_{33} \xi \mid v_{33}^{*} \eta\right)=\left(v_{33} c_{33} \xi \mid \eta\right)=(\xi \mid \eta) .
$$

Since $D_{0}$ is a core of $v^{-1}, D \cap \Re_{3}$ is dense in $\Re_{3}$. Hence $R\left(v_{33}^{*}\right) \subset D\left(c_{33}^{*}\right)$. Since $R\left(v_{33}^{*}\right)^{\perp}=\operatorname{Ker} v_{33}=\{0\}, D\left(c_{33}^{*}\right)$ is dense in $\Re_{3}$ and hence $c_{33}$ is closable. Thus $v_{33} \bar{c}_{33}=1$ on $D\left(\bar{c}_{33}\right)$. Indeed, if $\xi \in D\left(\bar{c}_{33}\right)$, there exists a sequence $\left\{\xi_{n}: n \in N\right\}$ in $D\left(c_{33}\right)$ such that $\xi_{n} \rightarrow \xi$ and $c_{33} \xi_{n} \rightarrow \bar{c}_{33} \xi$. Thus

$$
v_{33} \bar{c}_{33} \xi=\lim _{n \rightarrow \infty} v_{33} c_{33} \xi_{n}=\lim _{n \rightarrow \infty} \xi_{n}=\xi
$$

so that $D\left(\bar{c}_{33}\right) \subset R\left(v_{33}\right)$. On the other hand, since $p_{3} v^{-1} \mid D_{0}=\left(0,0, c_{33}\right)$, it follows that $p_{3} v^{-1} \mid D_{0}$ is closable, $\left(p_{3} v^{-1} \mid D_{0}\right)^{-}=\left(0,0, \bar{c}_{33}\right)$ and

$$
D \subset D\left(\left(p_{3} v^{-1} \mid D_{0}\right)^{-}\right)=\Re_{1}+\Re_{2}+D\left(\bar{c}_{33}\right) .
$$

Combining this with (5.5), we have $R\left(v_{33}\right)=p_{3} D \subset D\left(\bar{c}_{33}\right)$. Consequently, $R\left(v_{33}\right)$ $=D\left(\bar{c}_{33}\right)$.

(ii) $\rightarrow(\mathrm{i})$ : Since $c_{31}=0, c_{32}=0$ and $c_{33}$ is closable by assumption, it follows 
from $R(v) \subset D\left(\left(p_{3} v^{-1} \mid D_{0}\right)^{-}\right)$that

$$
p_{3}=\left(p_{3} v^{-1} \mid D_{0}\right)^{-} v p_{3}=\left(0,0, \bar{c}_{33}\right) v p_{3}=\left(0,0, \bar{c}_{33} v_{33}\right) .
$$

Therefore $R\left(\bar{c}_{33}\right)=D\left(v_{33}\right)=\Re_{3}$ and $\bar{c}_{33} v_{33}=1$ on $\mathfrak{\Re}_{3}$. Consequently, we have $\operatorname{Ker} v_{33}=\{0\}$.

Q.E.D.

LEMMa 5.2. Let $u$ be a bounded \#-unitary whose point spectrum does not contain 1 , and $v=u-1$. Let $p$ be a neutral projection invariant under $v$ and $v^{\#}$, and $p_{1}=p, p_{2}=1-p-p^{\#}, p_{3}=p^{\#}$. Let us make the same assumption for $v$ and $\Re_{i}=p_{i} \Re(i=1,2,3)$ as in Lemma 5.1. Then the following two conditions are equivalent:

(i) $R\left(v_{11}\right)$ is dense in $\Re_{1}$; and

(ii) $c_{21}=0, c_{31}=0$ and $c_{11}$ is closable.

In this case, $\bar{c}_{11}=v_{11}^{-1}$.

Proof. First we notice that $\left(v^{\#}\right)^{-1}=\left(v^{-1}\right)^{\#}$. Indeed, since $\left(v^{*}\right)^{-1}=\left(v^{-1}\right)^{*}$, we know that $\left(v^{\#}\right)^{-1}=\left(J v^{*} J\right)^{-1}=J\left(v^{*}\right)^{-1} J=J\left(v^{-1}\right)^{*} J=\left(v^{-1}\right)^{\#}$. Put $v_{i j}=p_{i} v \mid \AA_{j}$ and $J_{i j}=p_{i} J \mid \Re_{j}$. Then $v$ is represented in the form (5.3). Thus

$$
v^{\sharp}=\left(\begin{array}{ccc}
J_{13} u_{33}^{*} J_{31} & J_{13} v_{23}^{*} J_{22} & J_{13} v_{13}^{*} J_{13} \\
0 & J_{22} v_{22}^{*} J_{22} & J_{22} v_{12}^{*} J_{13} \\
0 & 0 & J_{31} v_{11}^{*} J_{13}
\end{array}\right) \quad \text { on } \AA \text {. }
$$

Since $D_{0}=D \cap \Re_{1}+D \cap \Re_{2}+D \cap \Re_{3}\left(D=D\left(v^{-1}\right)\right)$ is a core of $v^{-1}$ by assumption, $v^{-1}$ is represented by $\left(c_{i j}\right)$ on $D_{0}$. Since $v^{\sharp}=(u-1)^{\sharp}=(1-u) u^{-1}$, it follows that $\left(v^{\sharp}\right)^{-1}=-u v^{-1}$. Therefore $D_{0}$ is also a core of $\left(v^{\#}\right)^{-1}$ and

$$
\left(v^{\sharp}\right)^{-1}=\left(\begin{array}{lll}
J_{13} c_{33}^{*} J_{31} & J_{13} c_{23}^{*} J_{22} & J_{13} c_{13}^{*} J_{13} \\
J_{22} c_{32}^{*} J_{31} & J_{22} c_{22}^{*} J_{22} & J_{22} c_{12}^{*} J_{13} \\
J_{31} c_{31}^{*} J_{31} & J_{31} c_{21}^{*} J_{22} & J_{31} c_{11}^{*} J_{13}
\end{array}\right) \quad \text { on } D_{0} .
$$

We now apply Lemma 5.1 to $v^{\sharp}$ in place of $v$. Then the following two conditions are equivalent:

(i) $\quad \operatorname{Ker} J_{31} v_{11}^{*} J_{13}=\{0\}$; and

(ii) $J_{31} c_{31}^{*} J_{31}=0, J_{31} c_{21}^{*} J_{22}=0$ and $J_{31} c_{11}^{*} J_{13} \mid D \cap \Re_{3}$ is closable.

It is clear that these conditions are equivalent to (i) and (ii), respectively. Furthermore, $c_{11}^{*}=\left(v_{11}^{*}\right)^{-1}$ and hence $\bar{c}_{11}=c_{11}^{* *}=v_{11}^{-1}$.

Q.E.D.

The above two lemmas tell us that, if $u$ is represented in the form of an upper triangular matrix by some neutral projection invariant under $u$ and $u^{\#}$ such that $R\left(u_{11}-1\right)^{-}=\Re_{1}$ and $\operatorname{Ker}\left(u_{33}-1\right)=\{0\}$, and if $D_{0}$ is a core of the inverse $(u-1)^{-1}$, then $(u-1)^{-1}$ is also represented by an upper triangular matrix 


$$
\left(\begin{array}{lll}
c_{11} & c_{12} & c_{13} \\
0 & c_{22} & c_{23} \\
0 & 0 & c_{33}
\end{array}\right) \text { and } \bar{c}_{i i}=\left(u_{i i}-1\right)^{-1} \text {. }
$$

This fact will be used in the following theorem:

Theorem 5.3. $A$ \#-selfadjoint operator $h$ in a Pontrjagin space is represented by a canonical triangular matrix for some selfdual Hilbertian inner product. In this case, $h_{22}$ is \#-spectral.

Proof. We may assume that $\operatorname{dim} \AA^{+}<\infty$ and $\operatorname{dim} \AA^{-}=\infty$. On a Pontrjagin space $\mathrm{Sp}(h) \backslash \boldsymbol{R}$ is finite and so the complement is not empty. Let $u$ be the Cayley transform $(h-\bar{\lambda} 1)(h-\lambda 1)^{-1}$ of $h$ for some $\lambda \notin \operatorname{Sp}(h) \cup \boldsymbol{R}$. Then $u$ is a (bounded) \#-unitary with $u-J u J$ of finite rank. Therefore $u$ is represented by a canonical triangular matrix by Theorem 2.6. Let $p$ be a neutral projection used to form a canonical triangular matrix. Let $e_{1}=p+p^{\#}$ and $e_{2}=1-p-p^{\#}$. Then $e_{1}$ and $e_{2}$ are \#-projections. Decompose $e_{j}(j=1,2)$ into the difference $e_{j}^{+}-e_{j}^{-}$of a (uniformly) \#-positive \#-projection $e_{j}^{+}$and a uniformly \#-negative \#-projection $e_{j}^{-}$.

Put $e^{ \pm}=e_{1}^{ \pm}+e_{2}^{ \pm}$. Then $e^{+}$and $e^{-}$are a uniformly \#-positive and a uniformly \#-negative \#-projections such that $e^{+}+e^{-}=1$. Since $e^{ \pm}=a J^{ \pm} a^{-1}$ for some \#-unitary $a$, we may assume that $e^{ \pm}=J^{ \pm}$for some selfdual Hilbertian inner product.

On the Pontrjagin space $\operatorname{dim} p=\operatorname{dim} p^{*}<\infty$. Let $n=\operatorname{dim} e_{1}^{-}$. The subspace $e_{1} \Re$ contains an $n$-dimensional (uniformly) \#-negative subspace. Since $D(h)$ is dense in $\AA$, we can select, by the subsequent Lemma 5.4, the subspace as a subspace of $D(h)$, which we denote by $\mathfrak{M}_{-}^{-}$. There exists then a \#-projection $f_{1}^{-}$onto $\mathfrak{M}_{1}^{-}$. Since $\mathfrak{M}_{1}^{-} \subset D(h)$, we have

$$
D(h)=\mathfrak{M}_{1}^{-}+\left\{D(h) \cap\left(1-f_{1}^{-}\right) \Re\right\}
$$

and $D(h) \cap\left(1-f_{1}^{-}\right) \Re$ is dense in $\left(1-f_{1}^{-}\right) \Re$. Let $m=\operatorname{dim} e^{+}$. Since $\left(1-f_{1}^{-}\right) \Re$ is infinite dimensional, we can choose an $m$-dimensional (uniformly) \#-positive subspace $\mathfrak{M}^{+}$of $D(h) \cap\left(1-f_{1}^{-}\right) \Re$. There exists a \#-projection $f^{+}$onto $\mathfrak{M}^{+}$. It is clear from the above construction that the sum $f^{+}+f_{1}^{-}$is a \#-projection. Since $\mathfrak{M}^{+} \subset D(h) \cap\left(1-f_{1}^{-}\right) \mathfrak{R}$, we have

$$
D(h)=\left(f^{+}+f_{1}^{-}\right) \Re+\left\{D(h) \cap\left(1-f^{+}-f_{1}^{-}\right) \Re\right\} .
$$

Since \#-projections $e^{+}+e_{1}^{-}$and $f^{+}+f_{1}^{-}$are finite dimensional and since $\operatorname{dim} e^{+}$ $=\operatorname{dim} f^{+}$and $\operatorname{dim} e_{1}^{-}=\operatorname{dim} f_{1}^{-}$, we can find a bounded $\#$-unitary $w$ such that

$$
w f^{+} w^{\#}=e^{+} \quad \text { and } \quad w f_{1}^{-} w^{\#}=e_{1}^{-} .
$$


Indeed, since (uniformly) \#-positive \#-projections $e^{+}$and $f^{+}$are maximal nonnegative, there exists a bounded $\#$-unitary $w_{1}$ such that $w_{1} f^{+} w_{1}^{\#}=e^{+}$. Put

$$
\left(e_{1}^{-}\right)^{\prime}=w_{1} f_{1}^{-} w_{1}^{\#} \quad \text { and } \quad\left(e_{2}^{-}\right)^{\prime}=w_{1}\left(1-f^{+}-f_{1}^{-}\right) w_{1}^{\#} .
$$

Then \#-projections $\left(e_{1}^{-}\right)^{\prime}$ and $\left(e_{2}^{-}\right)^{\prime}$ are uniformly \#-negative such that $\operatorname{dim}\left(e_{1}^{-}\right)^{\prime}$ $=\operatorname{dim} e_{1}^{-}$and $\left(e_{1}^{-}\right)^{\prime}+\left(e_{2}^{-}\right)^{\prime}=e^{-}$. Since $e^{ \pm}=J^{ \pm}, e^{-} \AA$ is a Hilbert space with respect to the inner product $\xi, \eta \rightarrow-\langle\xi, \eta\rangle=(\xi \mid \eta)$. There exists then a unitary $w_{2}^{\prime}$ on $e^{-} \Omega$ such that $w_{2}^{\prime}\left(e_{1}^{-}\right)^{\prime} w_{2}^{\prime-1}=e_{1}^{-}$. Denote by $w_{2}$ the extension of $w_{2}^{\prime}$ to $\AA$ obtained by defining 1 on $e^{+} \Re$. Then $w_{2}$ is a bounded \#-unitary such that

$$
w_{2}\left(e_{1}^{-}\right)^{\prime} w_{2}^{\sharp}=e_{1}^{-} \quad \text { and } \quad w_{2}\left(e_{2}^{-}\right)^{\prime} w_{2}^{\sharp}=e_{2}^{-} .
$$

It is easy to see that $w=w_{2} w_{1}$ is a bounded \#-unitary with (5.8). Consequently, whw $w^{\#}$ is \#-selfadjoint and

$$
\begin{aligned}
D\left(w h w^{\sharp}\right) & =\left(e^{+}+e_{1}^{-}\right) \Re+\left\{D\left(w h w^{\#}\right) \cap e_{2}^{-} \Re\right\} \\
& =p \Re+\left\{D\left(w h w^{\sharp}\right) \cap\left(1-p-p^{\#}\right) \Re\right\}+p^{\#} \Re
\end{aligned}
$$

by (5.7).

From these discussions we may assume that $D(h)$ is of the form $p \mathfrak{i}+$ $\left\{D(h) \cap\left(1-p-p^{\sharp}\right) \Re\right\}+p^{\sharp} \Re$ for some selfdual Hilbertian inner product. Thus we can apply Lemmas 5.1 and 5.2 to $v=u-1$. Since $\operatorname{dim} p=\operatorname{dim} p^{\#}<\infty$ and $D(h)=D\left(v^{-1}\right)$, conditions (i) in Lemmas 5.1 and 5.2 hold. Therefore the inverse of $u-1$ is represented by an upper triangular matrix (5.3). Hence the Cayley transform $h=(\lambda u-\bar{\lambda} 1)(u-1)^{-1}$ is also represented by an upper triangular matrix. In particular

$$
h_{22}=\left(\lambda u_{22}-\bar{\lambda} 1\right)\left(u_{22}-1\right)^{-1} .
$$

Since $u_{22}$ is \#-spectral in $\Re_{2}, h_{22}$ is also \#-spectral.

Q.E.D.

Perturbing a finite dimensional \#-positive subspace slightly, we may assume it to be contained in a given dense subspace as follows:

LEMma 5.4. Let $D$ be a dense subspace of $\{\Omega,\langle\rangle$,$\} . For each n \in N$ (with $n \leq \operatorname{dim} \AA^{+}$) there exists an $n$-dimensional \#-positive subspace (of $\Re$ ) contained in $D$.

Proof. Let $\mathfrak{M}$ be an $n$-dimensional \#-positive subspace of $\mathfrak{R}$. Since it is finite dimensional, it is uniformly \#-positive:

$$
\langle\xi, \xi\rangle \geq \alpha\|\xi\|^{2}, \quad \xi \in \mathfrak{M}
$$

for some $\alpha>0$.

Let $\varepsilon$ be any positive number. For an orthonormal basis $\left\{\xi_{1}, \cdots, \xi_{n}\right\}$ of 
$\mathfrak{M}$ we choose a subset $\left\{\xi_{1}^{\prime}, \cdots, \hat{\xi}_{n}^{\prime}\right\}$ of $\Re$ satisfying that $\xi_{j}+\xi_{j}^{\prime} \in D$ and $\left\|\xi_{j}^{\prime}\right\|<\varepsilon$ for $j=1, \cdots, n$. If $\varepsilon$ is sufficiently small, then the linear span $\mathfrak{N}$ of $\left\{\xi_{1}+\xi_{1}^{\prime}\right.$, $\left.\cdots, \xi_{n}+\xi_{n}^{\prime}\right\}$ is an $n$-dimensional subspace contained in $D$. In order to show this fact, we consider an $n \times n$ matrix $a=\left(\alpha_{i j}\right)$ defined by $\alpha_{i j}=\left(\xi_{j}^{\prime} \mid \xi_{i}\right)$. If $\varepsilon$ is so small that $\|a\|<1$, then $b=1+a$ is invertible. Thus, if $\sum_{j=1}^{n} \mu_{j}\left(\xi_{j}+\xi_{j}^{\prime}\right)=0$ for $\mu_{j} \in C$, then

$$
b\left(\begin{array}{c}
\mu_{1} \\
\vdots \\
\mu_{n}
\end{array}\right)=0
$$

and hence $\mu_{1}=\cdots=\mu_{n}=0$.

Now, let $\xi$ be an arbitrary vector in $\Re$. It is of the form $\sum_{j=1}^{n} \lambda_{j}\left(\xi_{j}+\xi_{j}^{\prime}\right)$ for some $\lambda_{j} \in C$. Then

$$
b\left(\begin{array}{c}
\lambda_{1} \\
\vdots \\
\lambda_{n}
\end{array}\right)=\left(\begin{array}{c}
\left(\xi \mid \xi_{1}\right) \\
\vdots \\
\left(\xi \mid \xi_{n}\right)
\end{array}\right)
$$

and so $\left|\lambda_{i}\right| \leq\left(\sum_{j=1}^{n}\left|\lambda_{j}\right|^{2}\right)^{1 / 2} \leq\left\|b^{-1}\right\|\|\xi\|$. Hence

$$
\sum_{j=1}^{n}\left|\lambda_{j}\right| \leq n\left\|b^{-1} \mid\right\| \xi \|
$$

and

$$
\left|\|\xi\|-\left\|\sum_{j=1}^{n} \lambda_{j} \xi_{j}\right\|\right| \leq\left\|\xi-\sum_{j=1}^{n} \lambda_{j} \xi_{j}\right\| \leq n \varepsilon\left\|b^{-1}\right\|\|\xi\| \cdot
$$

If $\varepsilon$ is sufficiently small and less than $1 /\left(n\left\|b^{-1}\right\|\right)$, then

$$
\begin{aligned}
\langle\xi, \xi\rangle & \geq\left\langle\sum_{j=1}^{n} \lambda_{j} \xi_{j}, \sum_{k=1}^{n} \lambda_{k} \xi_{k}\right\rangle-3 \varepsilon\left(\sum_{j=1}^{n}\left|\lambda_{j}\right|\right)^{2} \\
& \geq \alpha\left\|\sum_{j=1}^{n} \lambda_{j} \xi_{j}\right\|^{2}-3 \varepsilon\left(\sum_{j=1}^{n}\left|\lambda_{j}\right|\right)^{2} \\
& \geq\left\{\alpha\left(1-n \varepsilon\left\|b^{-1}\right\|\right)^{2}-3 \varepsilon\left(n\left\|b^{-1}\right\|\right)^{2}\right\}\|\xi\|^{2},
\end{aligned}
$$

where $\alpha$ is a positive constant due to the (uniform) \#-positivity of $\mathfrak{M}$. Therefore if $\varepsilon$ is less than both $1 /\left(n\left\|b^{-1}\right\|\right)$ and $\alpha /\left(2 n\left\|b^{-1}\right\|+3 n^{2}\left\|b^{-1}\right\|^{2}\right)$, then the coefficient of $\|\xi\|^{2}$ of the right hand side is positive. Thus $\mathfrak{N}$ is an $n$-dimensional (uniformly) \#-positive subspace contained in $D$.

Q.E.D.

We want to generalize the above theorem to a Krein space. But, unfortunately, it will turn out to be clear from the following examples that the method of the Cayley transforms does not work for this general case without assuming that $R\left(u_{11}-1\right)^{-}=\Re_{1}$ and $\operatorname{Ker}\left(u_{33}-1\right)=\{0\}$. 
In the sequel we shall discuss such examples. As a result, we will be able to give an example of a bounded \#-unitary such that $R\left(u_{11}-1\right)^{-} \subseteq \Omega_{1}$ and $\operatorname{Ker}\left(u_{33}-1\right) \neq\{0\}$. Our main discussion will start from Example 2. Examples 1 and $1^{\prime}$ are prepared only to give examples of operators represented by an upper triangular matrix whose inverse is not upper triangular.

EXAMPLE 1. Let $v$ be a bounded operator represented by an upper triangular $3 \times 3$ matrix $\left(v_{i j}\right)$ of operators such that

$v_{11}$ is an isometry on $\Re_{1}, v_{22}$ is a unitary, $v_{33}$ is a coisometry on $\Re_{3}$,

$v_{12}=0, v_{23}=0$,

$v_{13}$ is a partial isometry with $v_{11} v_{11}^{*}+v_{13} v_{13}^{*}=1_{\Omega_{1}}$ and $v_{13}^{*} v_{13}+v_{33}^{*} v_{33}=v_{\Omega_{3}}$.

Then $v_{13} v_{33}^{*}=0$ and $v_{11}^{*} v_{13}=0$. Thus $v$ is a unitary and hence the matrix representation of $v^{-1}=v^{*}$ is not upper triangular.

EXAMPLE $1^{\prime}$. Let $v=\left(v_{i j}\right)$ be a bounded operator represented by an upper triangular matrix satisfying the following:

$v_{11}$ is an isometry on $\Omega_{1}, v_{33}$ is a coisometry on $\Omega_{3}$,

$v_{12}, v_{13}, v_{22}$ and $v_{23}$ are partial isometries with

$$
\begin{aligned}
& v_{12}^{*} v_{12}+v_{22}^{*} v_{22}=1_{\Omega_{2}}, \quad v_{13}^{*} v_{13}+v_{23}^{*} v_{23}+v_{33}^{*} v_{33}=1_{\Omega_{3}}, \\
& v_{11} v_{11}^{*}+v_{12} v_{12}^{*}+v_{13} v_{13}^{*}=1_{\Omega_{1}}, \quad v_{22} v_{22}^{*}+v_{23} v_{23}^{*}=1_{\Omega_{2}} .
\end{aligned}
$$

Then $v_{22} v_{12}^{*}=0, v_{23} v_{13}^{*}=0, v_{33} v_{13}^{*}=0, v_{33} v_{23}^{*}=0, v_{12}^{*} v_{11}=0, v_{13}^{*} v_{11}=0, v_{13}^{*} v_{12}=0$ and $v_{23}^{*} v_{22}$ $=0$. Thus $v$ is a unitary whose inverse $v^{-1}$ is not represented by an upper triangular matrix.

In the following example, $p$ denotes a neutral projection and $p_{1}=p, p_{2}=$ $1-p-p^{\sharp}, p_{3}=p^{\sharp}, \mathfrak{\Re}_{j}=p_{j} \mathfrak{R}, J_{i j}=p_{i} J \mid \Re_{j}$.

EXAMPLE 2. Let $u_{11}$ be a bounded operator in $\Re_{1}$ such that

a) $u_{11}$ has a bounded inverse with domain $\Omega_{1}$, and

b) 1 is in the residual spectrum of $u_{11}$ and the range $R\left(u_{11}-1\right)$ is closed. Put $u_{33}=\left(J_{31} u_{11}^{*} J_{13}\right)^{-1}$ and $u_{13}=i\left(u_{11}^{*}\right)^{-1} J_{13}\left(=i J_{13} u_{33}\right)$. Let $u_{22}$ be a bounded \#-unitary on $\Omega_{2}$ with $1 \notin \mathrm{Sp}\left(u_{22}\right)$. Then

$$
u=\left(\begin{array}{ccc}
u_{11} & 0 & u_{13} \\
0 & u_{22} & 0 \\
0 & 0 & u_{33}
\end{array}\right)
$$

is a bounded \#-unitary on $\mathscr{R}=\Re_{1}+\Re_{2}+\Re_{3}$ such that

(i) 1 is not in the point spectrum of $u$, and

(ii) $R(u-1)$ is of the form $\Re_{1}+\Re_{2}+\left\{R(u-1) \cap \Re_{3}\right\}$ and dense in $\Re$. 
Proof. The boundedness of $u$ is clear. Since

$$
u^{\#}=\left(\begin{array}{ccc}
J_{13} u_{33}^{*} J_{31} & 0 & J_{13} u_{13}^{*} J_{13} \\
0 & J_{22} u_{22}^{*} J_{22} & 0 \\
0 & 0 & J_{31} u_{11}^{*} J_{13}
\end{array}\right),
$$

$u u^{\sharp}=u^{\sharp} u=1$ by direct computation. Thus $u$ is a bounded \#-unitary.

(i) If $u \xi=\xi$ for some $\xi=\xi_{1}+\xi_{2}+\xi_{3}$ with $\xi_{j} \in \Re_{j}(j=1,2,3)$, then

$$
u_{11} \xi_{1}+u_{13} \xi_{3}=\xi_{1}, \quad u_{22} \xi_{2}=\xi_{2}, \quad u_{33} \xi_{3}=\xi_{3} .
$$

Since $1 \notin \operatorname{Sp}\left(u_{22}\right)$ by assumption, $\xi_{2}=0$. Since $\Re_{1} \ominus R\left(y_{11}-1\right)=\operatorname{Ker}\left(u_{11}^{*}-1\right)$, $J_{31} u_{11}^{*} J_{13}=u_{33}^{-1}$ and $u_{33}^{-1}-1=u_{33}^{-1}\left(1-u_{33}\right)$, it follows that

$$
\begin{aligned}
J_{31}\left\{\Re_{1} \ominus R\left(u_{11}-1\right)\right\} & =J_{31} \operatorname{Ker}\left(u_{11}^{*}-1\right)=\operatorname{Ker}\left(J_{31} u_{11}^{*} J_{13}-1\right) \\
& =\operatorname{Ker}\left(u_{33}^{-1}-1\right)=\operatorname{Ker}\left(u_{33}-1\right) .
\end{aligned}
$$

Thus $J_{13} \xi_{3} \in \Re_{1} \ominus R\left(u_{11}-1\right)$ and $\left(u_{11}^{*}\right)^{-1}=1$ on $\Re_{1} \ominus R\left(u_{11}-1\right)$. Therefore

$$
-\left(u_{11}-1\right) \xi_{1}=u_{13} \xi_{3}=i\left(u_{11}^{*}\right)^{-1} J_{13} \xi_{3}=i J_{13} \xi_{3},
$$

which belongs to both $R\left(u_{11}-1\right)$ and $\Omega_{1} \ominus R\left(u_{11}-1\right)$. Thus $\left(u_{11}-1\right) \xi_{1}=0$ and $\xi_{3}=0$. Since 1 belongs to the residual spectrum of $u_{11}$, it does not belong to the point spectrum and hence $\xi_{1}=0$. Consequently, $\xi=0$.

(ii) Let $v=u-1$ and $D=R(u-1)$. Then, by virtue of (5.4),

$$
D \cap \Re_{1}=R\left(v_{11}\right)+v_{13} \operatorname{Ker} v_{33}, \quad D \cap \Re_{2}=v_{22} \Re_{2} \quad \text { and } \quad D \cap \Re_{3}=v_{33} \Re_{3} .
$$

Since $R\left(u_{11}-1\right)$ is closed and

$$
\begin{aligned}
v_{13} \operatorname{Ker} v_{33} & =i\left(u_{11}^{*}\right)^{-1} J_{13} \operatorname{Ker}\left\{\left(J_{31} u_{11}^{*} J_{13}\right)^{-1}-1\right\} \\
& =i\left(u_{11}^{*}\right)^{-1} \operatorname{Ker}\left\{\left(u_{11}^{*}\right)^{-1}-1\right\} \\
& =\operatorname{Ker}\left\{\left(u_{11}^{*}\right)^{-1}-1\right\} \\
& =\operatorname{Ker}\left(u_{11}^{*}-1\right)=\Re_{1} \ominus R\left(u_{11}-1\right),
\end{aligned}
$$

it follows that

$$
D \cap \Re_{1}=R\left(u_{11}-1\right)+\left\{\Re_{1} \ominus R\left(u_{11}-1\right)\right\}=\Re_{1} .
$$

Since $1 \notin \operatorname{Sp}\left(u_{22}\right)$ by assumption, we have

$$
D \cap \AA_{2}=\left(u_{22}-1\right) \AA_{2}=\AA_{2} \text {. }
$$

Thus $D$ contains $\Re_{1}+\Re_{2}$ and hence is decomposed into the sum $\Re_{1}+\Re_{2}+$ $\left(D \cap \Re_{3}\right)$. It is clear that 


$$
\begin{aligned}
D \cap \Re_{3} & =R\left(u_{33}-1\right)=J_{31}\left\{\left(u_{11}^{*}\right)^{-1}-1\right\} J_{13} \Re_{3} \\
& =J_{31}\left(u_{11}^{*}-1\right)\left(u_{11}^{*}\right)^{-1} J_{13} \Re_{3}=J_{31}\left(u_{11}^{*}-1\right) \Re_{1} .
\end{aligned}
$$

Since 1 is not in the point spectrum of $u_{11}, \Re_{1} \ominus\left\{R\left(u_{11}^{*}-1\right)^{-}\right\}=\operatorname{Ker}\left(u_{11}-1\right)=\{0\}$. Thus $R\left(u_{11}^{*}-1\right)$ is dense in $\Re_{1}$ and so $D \cap \Re_{3}$ is dense in $\Re_{3}$. Consequently, $D$ is dense in $\Re$.

Q.E.D.

EXAmPle 3. Using the bounded \#-unitary $u$ obtained by (5.9) in Example 2 , we set $v=u-1$. Since $R(u-1)$ is dense in $\Re$, the operator $v$ has the inverse with dense domain. Moreover, the inverse is not represented by an upper triangular $3 \times 3$ matrix of operators, contrary to $v$.

Proof. The matrix representation of $v^{-1}$ is of the form

$$
\left(\begin{array}{lcc}
c_{11} & 0 & c_{13} \\
0 & v_{22}^{-1} & 0 \\
c_{31} & 0 & c_{33}
\end{array}\right) .
$$

We shall show that $c_{31} \neq 0$. Since $D\left(v^{-1}\right)=R(u-1)$ is of the form $\Re_{1}+\Re_{2}+$ $\left\{R(v) \cap \Re_{3}\right\}$ by Example 2, we see that $c_{11}$ is bounded. Therefore, the $(1,1)$ components of the matrix representations corresponding to $v v^{-1}=1$ and $v^{-1} v$ $=1$ satisfy

$$
v_{11} c_{11}+v_{13} c_{31}=1 \quad \text { and } \quad c_{11} v_{11}=1 \text {, }
$$

respectively. By assumption, $R\left(v_{11}\right)$ is closed. From the second equality, $c_{11}$ is of the form $v_{11}^{-1} \circ p+c$ for some operator $c$ on $\Re_{1}$ with $c p=0$, where $p$ is the projection of $\Re_{1}$ onto $R\left(v_{11}\right)$. It follows from the first equality that

$$
v_{13} c_{31}=(1-p)-v_{11} c .
$$

Since 1 belongs to the residual spectrum of $u_{11}$, we see that $\Omega_{1} \ominus R\left(v_{11}\right) \neq\{0\}$ and so $1-p \neq 0$. Therefore $1-p-v_{11} c \neq 0$ and hence $c_{31} \neq 0$.

ExAmple 4. Now, we shall give an example of an operator which satisfies conditions a) and b) in Example 2. Let $S$ be the one sided shift on $\ell^{2}(N)$ defined by

$$
\left(\lambda_{1}, \lambda_{2}, \cdots\right) \longrightarrow\left(0, \lambda_{1}, \lambda_{2}, \cdots\right) \text {. }
$$

Then $x=1+(1 / 2) S$ is an invertible bounded operator whose inverse is bounded and defined everywhere. Since $x$ is injective and $R(x-1)=\left\{\left(\lambda_{i}\right) \in \ell^{2}(N)\right.$ : $\left.\lambda_{1}=0\right\}, 1$ belongs to the residual spectrum of $x$ and $R(x-1)$ is closed.

Using the similar argument as in the proof of Theorem 5.3 with the help 
of Theorems 2.6 and 4.4, Lemmas 5.1 and 5.2, we can obtain the following artificial theorem:

THEOREM 5.5. Let $h$ be a \#-selfadjoint operator satisfying that

a) $\AA^{+} \subset D$, where $D=D(h)$,

b) $J^{+} h J^{-}\left\{\left(J^{-} h \mid \Re^{-}\right)-\lambda_{0} 1\right\}^{-1}$ is compact for some $\lambda_{0} \notin \operatorname{Sp}\left(J^{-} h \mid \Re^{-}\right)$,

c) $D \cap \Re_{1}+D \cap \Re_{2}+D \cap \Re_{2}$ is a core of $h$, where $\Re_{j}=p_{j} \AA$ and $p_{j}(j=1,2,3)$ are projections of a canonical triangular matrix for the Cayley transform $u=$ $(h-\bar{\lambda} 1)(h-\lambda 1)^{-1}$ of $h$ at some $\lambda$ with $\left\|J^{ \pm} h J^{\mp}\right\|<|\operatorname{Im} \lambda|$.

If $R\left(u_{11}-1\right)^{-}=\Re_{1}$ and $\operatorname{Ker}\left(u_{33}-1\right)=\{0\}$, then $h$ is represented by a canonical triangular matrix $\left(h_{i j}\right)$ and coincides with $\left(h_{i j}\right)$ on the core in c). In this case, $h_{22}$ is quasi-\#-spectral.

\section{$\S 6$. One parameter group of bounded $\sharp$-unitaries}

We will consider the canonical triangular matrix for a strongly continuous one parameter group of bounded \#-unitaries. Before going into this statement, we will prepare Theorems 6.1 and 6.2, which are known. For the sake of completeness we will repeat these assertions.

Let $\{u(t): t \in R\}$ be a strongly continuous representation of $R$ by bounded $\#$-unitaries. Since $\|u(-t)\|=\|u(t)\|$ and $1 \leq\|u(t)\|\|u(-t)\|$, it follows that $\|u(t)\|$ $\geq 1$ for all $t$. By virtue of the general theory on a one parameter semi-group of bounded operators, there exist an $M \geq 1$ and a $\beta \in R$ such that $\|u(t)\| \leq$ $M \exp (\beta|t|)$ for all $t$. Therefore, in a Krein space, we see that

$$
1 \leq\|u(t)\| \leq M \exp (\beta|t|), \quad t \in \boldsymbol{R}
$$

for some $M \geq 1$ and $\beta \geq 0$.

THEOREM 6.1 ([9]). If $\{u, \Re\}$ is a strongly continuous representation of $\boldsymbol{R}$ by bounded \#-unitaries, then there exists a \#-selfadjoint operator $h$ with

$$
u(t)=\exp (i t h), \quad t \in \boldsymbol{R}
$$

In particular, $\xi \in D(h)$ if and only if the limit

$$
\lim _{t \downarrow 0}(1 / i t)\{u(t)-1\} \xi
$$

exists, and the value is $h \xi$.

Proof. According to the general theory on a one parameter semigroup of bounded operators, this theorem is already known except for the fact that $h$ is \#-selfadjoint. First we will show that the closed operator $h$ is \#-symmetric. For any $\xi$, $\eta$ in $D(h)$ we obtain 


$$
\begin{aligned}
\langle h \xi, \eta\rangle & =\lim _{t \downarrow 0}\langle(1 / i t)\{u(t)-1\} \xi, \eta\rangle \\
& =\lim _{t \downarrow 0}\langle u(t) \xi,(1 / i t)\{u(t)-1\} \eta\rangle=\langle\xi, h \eta\rangle .
\end{aligned}
$$

Next we suppose that $M \geq 1$ and $\beta \geq 0$ satisfy $\|u(t)\| \leq M \exp (\beta|t|)$ for all $t$. For any $t>\beta$ the closed operator $t 1-i$ h has the bounded inverse and

$$
(t 1-i h)^{-1} \hat{\xi}=\int_{0}^{\infty}\{\exp (-t s)\} u(s) \xi d s, \quad \xi \in \mathfrak{A} .
$$

Indeed, if $t>\beta$ and $\xi \in \Re$, then

$$
\left\|(t 1-i h)^{-1} \xi\right\| \leq M\left(\int_{0}^{\infty} \exp (-(t-\beta) s) d s\right)\|\xi\|=\frac{M}{t-\beta}\|\xi\| .
$$

Thus $(h+i t 1) D(h)=\Re$. Replacing $u(s)$ in the above integrand by $u(-s)=$ $u(s)^{\#}$, we also have $(h-i t 1) D(h)=\Omega$. Therefore the Cayley transform $(h-i t 1)$ $\times(h+i t 1)^{-1}$ is a bounded $\#$-unitary. Consequently, $h$ is $\#$-selfadjoint. Q.E.D.

REMARK. In the above theorem the representation $\{u, \Re\}$ is norm continuous if and only if $h$ is bounded.

Among \#-selfadjoint operators there exists an operator whose spectrum is the whole complex plane as shown at the beginning of Section 4 . In this case, the inequality $\|\exp (i t h)\| \leq M \exp (\beta|t|)$ does not hold. Therefore we have a problem: when does a \#-skewadjoint operator $i$ turn out to be a generator? For this we remark the following inequality: if $t>\beta$, then

$$
\left\|(t 1-i h)^{-m}\right\| \leq M(t-\beta)^{-m}, \quad m \in N .
$$

For $m=1$ we have already shown this in the above proof. For $m>1$, if $t>\beta$ and $\xi \in \Re$ we have

$$
\begin{aligned}
\left\|(t 1-i h)^{-m} \xi\right\| & =\left\|\frac{(-1)^{m-1}}{(m-1) !}\left(\frac{d}{d t}\right)^{m-1}(t 1-i h)^{-1} \xi\right\| \\
& =\left\|\frac{(-1)^{m-1}}{(m-1) !}\left(\frac{d}{d t}\right)^{m-1} \int_{0}^{\infty}\{\exp (-t s)\} u(s) \xi d s\right\| \\
& \leq \frac{1}{(m-1) !}\left(\int_{0}^{\infty}(-s)^{m-1} \exp (-(t-\beta) s) d s\right)\|\xi\| \\
& =M(t-\beta)^{-m}\|\xi\| .
\end{aligned}
$$

For any \#-selfadjoint operator in a Pontrjagin space, there always exist an $M \geq 1$ and a $\beta \geq 0$ which satisfy inequality (6.1).

Theorem 6.2 ([9]). Let $h$ be a \#-selfadjoint operator. Then ih is a gener- 
ator of a strongly continuous representation of $\boldsymbol{R}$ by bounded \#-unitaries if and only if there exist an $M \geq 1$ and $a \beta \geq 0$ such that the operator $t 1$ - ih has $a$ bounded inverse satisfying (6.1) for all $t>\beta$.

The proof is completed by an argument analogous to the one for a one parameter semi-group of bounded operators in a Banach space, [13, 17].

In the following theorem we will give a characterization for a strongly continuous \#-unitary representation to be \#-spectral.

TheOREM 6.3. Let $G$ be an amenable group and $\{u, \Re\}$ a strongly continuous representation of $G$ by bounded \#-unitaries. Then the following two conditions are equivalent:

(i) $\sup \{\|u(t)\|: t \in G\}<\infty$; and

(ii) there exist a bounded \#-unitary $v$ and strongly continuous unitary representations $\left\{u^{ \pm}, \Re^{ \pm}\right\}$such that

$$
v u(t) u^{\sharp}=\left(\begin{array}{cc}
u^{+}(t) & 0 \\
0 & u^{-}(t)
\end{array}\right), \quad t \in G .
$$

This theorem is an immediate consequence of the stability of bounded \#-unitaries, $[1,8,13]$.

Proof. The implication (ii) $\rightarrow$ (i) is obvious. Suppose that (i) holds. First we notice that $S z$-Nagy's theorem is easily generalized from $Z$ to an amenable group $G$ as follows:

A strongly continuous \#-unitary representation $\{u, \Re\}$ of $G$ with $\sup \{\|u(t)\|: t \in G\}<\infty$ is similar to a strongly continuous unitary representation of $G$.

Now, let $a$ be a bounded operator with bounded inverse which implements the similarity, so that $w(t)=a^{-1} u(t) a$ is unitary. Since $u(t)$ is \#-unitary,

$$
\begin{aligned}
J & =u(t)^{*} J u(t)=\left(a w(t) a^{-1}\right) * J a w(t) a^{-1} \\
& =\left(a^{*}\right)^{-1} w(t) * a^{*} J a w(t) a^{-1} .
\end{aligned}
$$

Here we set $K=a^{*} J a$. Then $K$ is a bounded selfadjoint operator with bounded inverse, and commutes with $w(t)$. Let $p^{+}$and $p^{-}$be the spectral projections of $K$ corresponding to the intervals $(-\infty, 0)$ and $(0, \infty)$, respectively. Since $K$ has the bounded inverse, $p^{+}+p^{-}=1$. Put $e^{ \pm}=a p^{ \pm} a^{-1}$. Then $e^{ \pm}$are idempotents with $e^{+}+e^{-}=1$. Since

$$
\left\langle e^{+} \xi, e^{-} \eta\right\rangle=\left(K p^{+} a^{-1} \xi \mid p^{-} a^{-1} \eta\right)=0
$$

and

$$
\left\langle e^{ \pm} \xi, e^{ \pm} \xi\right\rangle=\left(K p^{ \pm} a^{-1} \xi \mid p^{ \pm} a^{-1} \xi\right)
$$


we find that $e^{ \pm}$are \#-projections and that $e^{+}$is \#-positive and $e^{-}$is \#-negative. Therefore there exists a bounded \#-unitary $v$ such that $v e^{ \pm} v^{\sharp}=J^{ \pm}$. Since $K$ commutes with $w(t)$, it follows that $e^{ \pm}$commutes with $u(t)$ and hence $J^{ \pm}$commutes with $v u(t) v^{\#}$. Thus the desired formula (6.2) is obtained. Q.E.D.

COROLLARY 6.4. Let $h$ be $a$ \#-selfadjoint operator. The following three conditions are equivalent:

(i) $h$ is \#-spectral;

(ii) $\sup \{\| \exp ($ ith $) \|: t \in R\}<\infty$ (i.e. $t \rightarrow \exp ($ ith $)$ is \#-spectral); and

(iii) the Cayley transform $(h-\bar{\lambda} 1)(h-\lambda 1)^{-1}$ for some $\lambda \notin \operatorname{Sp}(h) \cup \boldsymbol{R}$ is \#spectral.

CoRollary 6.5. Let $G$ be a compact group. If $u$ is a strongly continuous representation of $G$ by bounded \#-unitaries, then there exists a selfdual Hilbertian inner product such that $u(t)=u^{+}(t) \oplus u^{-}(t)$, where $u^{ \pm}$are strongly continuous unitary representations.

Proof. If $G$ is compact, then $\{\|u(t) \xi\|: t \in G\}$ is bounded for each $\xi$, and so $\sup _{t \in G}\|u(t)\|<\infty$ by the uniform boundedness theorem. Thus we can apply Theorem 6.3 to this case.

Q.E.D.

Finally we will give canonical triangular matrix representations for these strongly continuous representation of $R$ by bounded $\#$-unitaries. The following theorem is a consequence of Theorems 3.1 and 5.3.

THEOREM 6.6. Let $\{u, \Re\}$ be a strongly continuous representation of $\boldsymbol{R}$ by bounded \#-unitaries and ih the generator. If $u$ is norm continuous and $h-$ JhJ is compact, or if $\Re$ is a Pontrjagin space with $\Re^{+} \subset D(h)$, then $u(t)$ is represented by a canonical triangular matrix

$$
\left(\begin{array}{ccc}
u_{11}(t) & * & * \\
0 & u_{22}(t) & * \\
0 & 0 & u_{33}(t)
\end{array}\right)
$$

such that $u_{j j}(j=1,2,3)$ are strongly continuous representations of $\boldsymbol{R}$. Further, $u_{11}$ and $u_{33}$ act on netural subspaces and $u_{22}$ is a \#-unitary representation. In particular, if $u$ is norm continuous, then $u_{22}$ is quasi-\#-spectral and if $\Re$ is a Pontrjagin space, then $u_{22}$ is \#-spectral,

Proof. By Theorem 6.1, $u(t)$ is expressed in the form exp (ith) for some $\#$-selfadjoint operator $h$.

First we consider the case where $u$ is norm continuous and $h-J h J$ is compact. The norm continuity of $u$ implies the boundedness of $h$. Thus we 
can apply Theorem 3.1 to $h$ to obtain a canonical triangular matrix $\left(h_{i j}\right)$. L $\in i$ $p_{j}(j=1,2,3)$ be projections used to set up the matrix. Denote by $u_{j j}(t)$ the restriction of $p_{j} u(t)$ to $p \AA$. Then

$$
u_{j j}(t)=\exp \left(i t h_{1 j}\right) \quad \text { for } j=1,2,3 .
$$

Indeed, the equalities $p_{j} h^{n} p_{j}=\left(p_{j} h p_{j}\right)^{n}$ for all $n \in N$ imply

$$
\begin{aligned}
p_{j} u(t) p_{j} & =p_{j}\left\{\sum_{n=0}^{\infty} \frac{1}{n !}(i t h)^{n}\right\} p_{j} \\
& =\sum_{n=0}^{\infty} \frac{1}{n !}\left(i t p_{j} h p_{j}\right)^{n}-\left(1-p_{j}\right) \\
& =\exp \left(i t p_{j} h p_{j}\right)-\left(1-p_{j}\right) .
\end{aligned}
$$

Therefore $u_{j j}(j=1,2,3)$ is a strongly continuous representation with the desired properties.

In the case where $\Re$ is a Pontrjagin space with $\Re^{+} \subset D(h)$, we can repeat the same argument as above by means of Theorem 5.3. Q.E.D.

In Theorem 6.6, the assumption $\Re^{+} \subset D(h)$ is not essential, because we can delete it by choosing a selfdual Hilbertian inner product in a Pontrjagin space suitably by means of Lemma 5.4. Furthermore, we notice that $h$-JhJ is compact if and only if $u(t)-J u(t) J$ is compact for all $t$.

REMARK. By a \#-unitary representation of a locally compact group $G$ we mean a strongly continuous representation of $G$ by bounded \#-unitaries. Two \#-unitary representations $\left\{\pi_{1}, \AA_{1}\right\}$ and $\left\{\pi_{2}, \AA_{2}\right\}$ are equivalent if there exists a linear bijection $v$ of $\left\{\Omega_{1},\langle,\rangle_{1}\right\}$ to $\left\{\Re_{2},\langle,\rangle_{2}\right\}$ with either $\langle v \xi, v \eta\rangle_{2}=$ $\langle\xi, \eta\rangle_{1}$ or $\langle v \xi, v \eta\rangle_{2}=-\langle\xi, \eta\rangle_{1}$ such that $v \pi_{1}(t)=\pi_{2}(t) v$ for all $t \in G$. A \#-unitary representation is said to be \#-irreducible if there exist no non trivial \#-projections commuting with the \#-unitary representation.

(i) If $G$ is compact, then any \#-irreducible \#-unitary representation is realized in either of the spaces $\Re^{ \pm}$by Corollary 6.5. Therefore the set of equivalence classes of \#-irreducible \#-unitary representations corresponds bijectively to the usual dual $G^{\wedge}$ of $G$. For example, if $G=T$, then the set of equivalence classes of \#-irreducible \#-unitary representations is identified with $Z$.

If $G$ is not compact, the situation changes drastically as shown in the following example.

(ii) Let $G=\boldsymbol{R}$. Using a standard representation of a \#-selfadjoint operator on a finite dimensional Krein space, we can show that the set of equivalence classes of finite dimensional \#-irreducible \#-unitary representations corresponds bijectively to the set $\left(C_{+} \times N\right) \cup(R \times N)$. where $C_{+}$is the upper 
half plane $\{z \in C: \operatorname{Im} z>0\}$. To see the \#-unitary representation concretely, we shall introduce notations. Let $1_{n}$ and $S_{n}$ be the identity and the nilpotent in $M_{n}(C)$ with $S_{n}=\left(s_{j k}\right), s_{j k}=\delta_{k-j, 1}$. Moreover, let $J_{n}$ be the matrix in $M_{n}(C)$ with $J_{n}=\left(a_{j k}\right), a_{j k}=\delta_{j+k, n+1}$.

For a real $\lambda$, \#-unitary representations $\pi_{\lambda, n}: t \rightarrow e^{i t \lambda} \exp \left(i t S_{n}\right)$ on $\left\{C^{n}, J_{n}\right\}$ are \#-irreducible. When $n=1, \pi_{\lambda, 1}$ is a character corresponding to $\lambda$ in the usual dual of $\boldsymbol{R}$. For a nonreal $\lambda \in \boldsymbol{C}_{+}$, a $\#$-unitary representation $\pi_{\lambda, n}$ :

$$
t \in \boldsymbol{R} \longrightarrow \exp \left\{i t\left(\begin{array}{cc}
\lambda 1_{n}+S_{n} & 0 \\
0 & \bar{\lambda} 1_{n}+S_{n}
\end{array}\right)\right\}
$$

on $\left\{C^{2 n}, J_{2 n}\right\}$ is \#-irreducible.

In order to consider infinite dimensional \#-irreducible \#-unitary representations we denote a two-sided shift on $\ell^{2}(Z)$ and one-sided shifts on $\ell^{2}(N)$ and $\ell^{2}(-N)$ by $S, S^{+}$and $S^{-}$, respectively:

$$
S \varepsilon_{n}=\varepsilon_{n+1}, \quad S^{ \pm} \varepsilon_{n}=\varepsilon_{n \pm 1},
$$

where $\left\{\varepsilon_{n}\right\}$ is the canonical basis for each of the spaces $\ell^{2}(Z), \ell^{2}(N)$ and $\ell^{2}(-N)$. Let $J$ be a unitary defined by $\left(J \varepsilon_{n} \mid \varepsilon_{m}\right)=\delta_{n+m, 0}$.

For a real $\lambda$, the \#-unitary representations $\pi_{\lambda}$ on $\left\{\ell^{2}(Z), J\right\}$ are $\#$-irreducible:

$$
\pi_{\lambda}(t)=e^{i t \lambda} \exp (i t S)
$$

For a nonreal $\lambda$, we have two different \#-irreducible \#-unitary representations. A \#-unitary representation $\pi_{\lambda}\left(\lambda \in C_{+}\right)$defined by

$$
\pi_{\lambda}(t)=\exp \left\{i t\left(\begin{array}{cc}
\lambda 1+S & 0 \\
0 & \bar{\lambda} 1+S
\end{array}\right)\right\} \quad \text { on }\left\{\ell^{2}(Z) \oplus \ell^{2}(Z),\left(\begin{array}{ll}
0 & J \\
J & 0
\end{array}\right)\right\}
$$

is \#-irreducible. To obtain another one we identify $\ell^{2}(Z \backslash\{0\})$ with a subspace of $\ell^{2}(Z)$ and denote by $p$ the projection onto $\ell^{2}(Z \backslash\{0\})$. The subspace $\ell^{2}(Z \backslash\{0\})$ is also identified with the direct sum $\ell^{2}(N) \oplus \ell^{2}(-N)$. A \#-unitary representation $\pi_{\lambda}^{0}(\lambda \in C)$ defined by

$$
\pi_{\lambda}^{0}(t)=\exp \left\{i t\left(\begin{array}{cc}
\lambda 1+S^{+} & 0 \\
0 & \bar{\lambda} 1+S^{-}
\end{array}\right)\right\}
$$

is \#-irreducible on $\left\{\ell^{2}(\boldsymbol{Z} \backslash\{0\}), p J \mid \ell^{2}(\boldsymbol{Z} \backslash\{0\})\right\}$.

We do not know whether any infinite dimensional \#-irreducible \#-unitary representation is equivalent to one of the above representations or not.

It will be important to investigate the $\#$-irreducible \#-unitary representations for non compact groups. 


\section{§ 7. Supplement for quasi-\# -spectrality}

We will discuss three examples of quasi-\#-spectral \#-selfadjoint operators. In the first example we will give a bounded \#-selfadjoint operator which preserves the spectrum. Modifying this operator slightly in the second example, we will have a bounded \#-selfadjoint operator which does not preserve the spectrum. The third example will treat the unbounded case.

EXAMPLE 1. Let $\mathfrak{S}$ be a Hilbert space and $\left\{\varepsilon_{n}: n \in \boldsymbol{Z}_{+}\right\}$an orthonormal basis. Define operators $x$ and $y$ on the linear span $D$ of the basis by

$$
x \varepsilon_{n}=(n+1) \varepsilon_{n} \quad \text { for } n \in Z_{+}
$$

and

$$
y \varepsilon_{n}=\left\{\begin{array}{ll}
0 & \text { for } n=0 \\
\varepsilon_{n-1} & \text { for } n \geq 1
\end{array} .\right.
$$

Then $x$ is symmetric and $y$ is a coisometry. Denote their closures by the same letters. It is easy to see that $x$ is positive selfadjoint and $D$ is its core.

LEMMA 7.1. Let $a$ be the restriction of

$$
1+\sum_{n=1}^{\infty} \frac{1}{n !} x^{n} y^{n}
$$

on $D$. Then

(i) $a D=D$ and $D \subset D\left(a^{*}\right)$;

(ii) $a$ is invertible and closable;

(iii) $a^{-1} x a=x(1+y)^{-1}$ and $a^{-1} x^{-1} a=(1+y) x^{-1}$ on $D$; and

(iv) $D$ is a core of $a^{*} \bar{a}$.

Proof. (i) Since $a \mid D_{0}$ is represented by an upper triangular matrix, we can verify that $a D=D$. Since each row of the matrix is an $\ell^{2}$ vector, $D\left(a^{*}\right)$ contains $D$.

(ii) Since $R(a)=a D=D$ is dense in $\mathfrak{S}$ and $a$ is injective, $a$ is invertible. Since $D\left(a^{*}\right)$ is dense in $\mathscr{S}$ by (i), $a$ is closable.

(iii) First we notice that $y y^{*}=1$ and $y^{*} x y=x-1$. We shall show

$$
x^{n+1} y^{n}=x^{n} y^{n} x-n x^{n} y^{n}, \quad n \in N
$$

on $D$ by mathematical induction. It is clear that, for $n=1$,

$$
x^{2} y=x y\left(y^{*} x y\right)=x y(x-1)=x y x-x y
$$

on $D$. Suppose that (7.2) holds for $n=k-1(k \geq 2)$. Then 


$$
\begin{aligned}
x^{k+1} y^{k} & =x\left(x^{k} y^{k-1}\right) y \\
& =x\left\{x^{k-1} y^{k-1} x-(k-1) x^{k-1} y^{k-1}\right\} y \\
& =x^{k} y^{k-1} x y-(k-1) x^{k} y^{k} \\
& =x^{k} y^{k}\left(y^{*} x y\right)-(k-1) x^{k} y^{k} \\
& =x^{k} y^{k}(x-1)-(k-1) x^{k} y^{k} \\
& =x^{k} y^{k} x-k x^{k} y^{k}
\end{aligned}
$$

on $D$. Accordingly, we have

$$
\begin{aligned}
x a & =x\left(1+\sum_{n=1}^{\infty} \frac{1}{n !} x^{n} y^{n}\right)=x+\sum_{n=1}^{\infty} \frac{1}{n !} x^{n+1} y^{n} \\
& =x+\sum_{n=1}^{\infty} \frac{1}{n !}\left(x^{n} y^{n} x-n x^{n} y^{n}\right) \\
& =\left(1+\sum_{n=1}^{\infty} \frac{1}{n !} x^{n} y^{n}\right) x-x\left(1+\sum_{n=1}^{\infty} \frac{1}{n !} x^{n} y^{n}\right) y \\
& =a x-x a y
\end{aligned}
$$

on $D$. Hence $x a(1+y)=a x$ on $D$. Thus we have $a^{-1} x a=x(1+y)^{-1}$ on $D$ and $a^{-1} x^{-1} a=(1+y) x^{-1}$ on $D$.

(iv) According to (i) we have $D \subset D\left(a^{*} \bar{a}\right)$. Put $\xi_{n}=a^{*} a \varepsilon_{n}$ for $n \in Z_{+}$. Then $a^{*} \bar{a}=\sum_{n=1}^{\infty} \xi_{n} \otimes \bar{\varepsilon}_{n}$ on $D$, where $\left(\xi_{n} \otimes \bar{\varepsilon}_{n}\right)(\eta)=\left(\eta \mid \varepsilon_{n}\right) \xi_{n}$. If $\eta \in D\left(a^{*} \bar{a}\right)$, then $a^{*} \bar{a} \eta=\sum_{n=1}^{\infty}\left(\eta \mid \varepsilon_{n}\right) \xi_{n}$. Put $\eta_{n}=\sum_{k=1}^{n}\left(\eta \mid \varepsilon_{k}\right) \varepsilon_{k}$ for $n \in N$. Then $\eta_{n} \in D, \eta_{n} \rightarrow \eta$ and

$$
a^{*} \bar{a} \eta_{n}=\sum_{k=1}^{n}\left(\eta \mid \varepsilon_{k}\right) \xi_{k} \longrightarrow \sum_{k=1}^{\infty}\left(\eta \mid \varepsilon_{k}\right) \xi_{k}=a^{*} \bar{a} \eta .
$$

Thus $D$ is a core of $a^{*} \bar{a}$.

Q.E.D.

Since $a D=D$ and $x D=D$, it follows that $a^{-1} x^{-1} a D=D$. From the above lemma we know that $a^{-1} x^{-1} a$ is bounded. Thus the closure of $a^{-1} x^{-1} a$ belongs to $\mathscr{L}(\mathfrak{E})$.

LEMMA 7.2. If $z$ is the closure of $a^{-1} x^{-1} a$, then

(i) $z D\left(a^{*} \bar{a}\right) \subset D\left(a^{*} \bar{a}\right)$; and

(ii) $z^{*} R\left(a^{*} \bar{a}\right) \subset R\left(a^{*} \bar{a}\right)$.

Proof. Notice that $z D=D$ and

$$
z^{*} a^{*} \bar{a}=a^{*} x^{*-1} a^{*-1} a^{*} \bar{a}=a^{*} x^{-1} \bar{a}=a^{*} \bar{a} \boldsymbol{z}
$$

on $D$. Since $D$ is a core of $a^{*} \bar{a}$ by (iv) in Lemma 7.1, if $\xi \in D\left(a^{*} \bar{a}\right)$, then there exists a sequence $\left\{\xi_{n} ; n \in N\right\}$ such that $\xi_{n} \rightarrow \xi$ and $a^{*} a \xi_{n} \rightarrow a^{*} \bar{a} \xi$. Then $z \xi_{n} \rightarrow z \xi$ and

$$
a^{*} \bar{a} z \xi_{n}=z^{*} a^{*} \bar{a} \xi_{n} \rightarrow z^{*} a^{*} \bar{a} \xi
$$


Thus $z \xi \in D\left(a^{*} \bar{a}\right)$ and $a^{*} \bar{a} z \xi=z^{*} a^{*} \bar{a} \xi$. This implies our desired results (i) and (ii).

Now we go back to the discussion on quasi-\#-spectrality. We define a Krein space $\{\Re, J\}$ by

$$
\Re=S \mathscr{C} \oplus \mathscr{\mathcal { C }} \text { and } J=\left(\begin{array}{ll}
0 & 1 \\
1 & 0
\end{array}\right) .
$$

Define operators in $\Re$ by

$$
h=z \oplus z^{*} \quad \text { and } \quad v=\bar{a} \oplus\left(a^{*}\right)^{-1} .
$$

Then $h$ is a bounded \#-selfadjoint operator and $v$ is a \#-unitary. Since

$$
D\left(v^{*} v\right)=D\left(a^{*} \bar{a}\right) \oplus R\left(a^{*} \bar{a}\right),
$$

it is invariant under $h$ by Lemma 7.2. Since

$$
v h v^{*}=x^{-1} \oplus x^{-1} \text { on } \quad v D\left(v^{*} v\right),
$$

$h$ is quasi-\#-spectral and $\operatorname{Sp}(h)=\operatorname{Sp}\left(\left(v h v^{\#} \mid D_{0}\right)^{-}\right)=\left\{n^{-1}: n \in N\right\}$. Indeed, if $\alpha_{0}$ $\neq 0$ and $\alpha_{k}=(-1)^{k}(k+1) ! \prod_{j=1}^{k}\left(j^{-1}-n^{-1}\right) \alpha_{0}$ for $k=1,2, \cdots, n-1$, then $\xi_{n}=$ $\sum_{k=0}^{n-1} \alpha_{k} \varepsilon_{k}$ satisfies $z \xi_{n}=n^{-1} \xi_{n}$. This shows that the quasi-\#-spectrality preserves the spectrum.

We shall perturb the above example slightly to obtain the following:

EXAMPLE 2. Let $a$ be the operator defined by (7.1). Using the operator $e_{10}=\varepsilon_{1} \otimes \bar{\varepsilon}_{0}$ of rank one given by $e_{10} \xi=\left(\xi \mid \varepsilon_{0}\right) \varepsilon_{1}$ for $\xi \in \mathfrak{S}$, we define an operator $b$ by $a+\alpha e_{10}$ for some $\alpha$ with $0<\alpha<1$. Since $\alpha \neq 1, b$ is injective and $b D=D$. By the same reason as for $a$, we see that $D \subset D\left(b^{*}\right)$ and $b$ is closable. Since

$$
b^{-1} x^{-1} b=\left(1+\alpha a^{-1} e_{10}\right)^{-1} a^{-1} x^{-1} a\left(1+\alpha a^{-1} e_{10}\right),
$$

the right hand side is then a product of three bounded operators. Hence $b^{-1} x^{-1} b$ is bounded. Denote the closure by $z^{\prime}$. Then

$$
k=z^{\prime} \oplus\left(z^{\prime}\right)^{*} \quad \text { and } \quad w=\bar{b} \oplus\left(b^{*}\right)^{-1}
$$

are a bounded $\#$-selfadjoint operator and a $\#$-unitary in $\{\AA, J\}$, respectively. It turns out by the same argument as in Lemma 7.2 that $k$ is quasi-\#-spectral with respect to $w$ :

$$
w k w^{\sharp}=x^{-1} \oplus x^{-1} \quad \text { on } \quad w D\left(w^{*} w\right) .
$$

The spectrum $\operatorname{Sp}\left(x^{-1} \oplus x^{-1}\right)$ is the set $\left\{n^{-1}: n \in N\right\}$, while $\operatorname{Sp}(k) \backslash \operatorname{Sp}\left(x^{-1} \oplus x^{-1}\right)$ is not empty as we can see in the following. 
To see the spectrum of $k=z^{\prime} \oplus\left(z^{\prime}\right)^{*}$, we must investigate the operator $z^{\prime}$, namely

$$
\left(b^{-1} x^{-1} b\right)^{-}=(1+c)^{-1}(1+y) x^{-1}(1+c),
$$

where $c=\alpha a^{-1} e_{10}$. Let $\alpha_{i j}=\left(a \varepsilon_{j} \mid \varepsilon_{i}\right)$ for $i, j \in Z_{+}$. Since $\left(a \varepsilon_{j} \mid \varepsilon_{i}\right)=0$ for $i>j, a$ is of the form $\sum_{i \leq j} \alpha_{i j} e_{i j}$, where $e_{i j}$ are matrix units $\varepsilon_{i} \otimes \bar{\varepsilon}_{j}$ for $i, j \in Z_{+}$. Since $\alpha_{00}=\alpha_{01}=\alpha_{11}=1$ and $\alpha_{10}=0$, we have $\left(a^{-1} \varepsilon_{0} \mid \varepsilon_{0}\right)=\left(a^{-1} \varepsilon_{1} \mid \varepsilon_{1}\right)=-\left(a^{-1} \varepsilon_{1} \mid \varepsilon_{0}\right)=1$ and $\left(a^{-1} \varepsilon_{0} \mid \varepsilon_{1}\right)=0$. Therefore $c=\alpha a^{-1} e_{10}=\alpha\left(-e_{00}+e_{10}\right)$, and hence the inverse $(1+c)^{-1}$ is of the form $1+d$, where $d=(\alpha-1)^{-1} c$. Indeed $(1+c)(1+d)=(1+d)(1+c)=$ $1+c+(\alpha-1)^{-1} c+(\alpha-1)^{-1} c^{2}=1$, for $c^{2}=-\alpha c$. Therefore

$$
\begin{aligned}
(1+c)^{-1}(1+y) x^{-1}(1+c) & =(1+y) x^{-1}+d(1+y) x^{-1}+(1+y) x^{-1} c+d(1+y) x^{-1} c \\
& =(1+y) x^{-1}+\{2(1-\alpha)\}^{-1}\left(\sum_{i, j=0}^{1} \beta_{i j} e_{i j}\right)
\end{aligned}
$$

where

$$
\beta_{00}=2-5 \alpha+2 \alpha^{2}, \quad \beta_{01}=1-3 \alpha, \quad \beta_{10}=3 \alpha-2 \alpha^{2}, \quad \beta_{11}=1 .
$$

Let $b$ be the $2 \times 2$ matrix $\left(\beta_{i j}\right)$. Since $\{2(1-\alpha)\}^{-1} \operatorname{Sp}(b) \subset \operatorname{Sp}\left(z^{\prime}\right)$, we have only to show that $\left\{(2(1-\alpha))^{-1} \mathrm{Sp}(b)\right\} \backslash \mathrm{Sp}\left(x^{-1}\right)$ is not empty. The proper equation of $b$ is given by $f(\lambda)=0$, where

$$
f(\lambda)=\lambda^{2}+(\alpha-3 / 2) \lambda+\left(-6 \alpha^{3}+13 \alpha^{2}-8 \alpha+2\right) / 4(\alpha-1)^{2} .
$$

Since the discriminant of this equation is given by

$$
4 \alpha^{4}+4 \alpha^{3}-15 \alpha^{2}+2 \alpha+1
$$

there exists an $\alpha_{0} \in(0,1)$ such that the equation $f(\lambda)=0$ has real solutions for $\alpha \in\left(0, \alpha_{0}\right]$ and nonreal solutions for $\alpha \in\left(\alpha_{0}, 1\right)$. For instance, if $\alpha=1 / 3$, then $\operatorname{Sp}(b)=\{1 / 4,3 / 4\}$. If $\alpha=1 / 2$, then $\operatorname{Sp}(b)=\{(1+2 i) / 2,(1-2 i) / 2\}$.

As indicated in Section 2, the definition of the quasi-\#-spectrality is not satisfactory for unbounded operators. The following example will be helpful when we consider this problem. An example of the similar type is also discussed in [2].

EXAMPLE 3. The set $\mathscr{S}(\boldsymbol{R})$ of rapidly decreasing functions on $\boldsymbol{R}$ is identified with a dense subset of $L^{2}(R)$, where $L^{2}(R)$ is the complex Hilbert space $L^{2}(\boldsymbol{R}, d t)$. Let $p$ and $q$ be selfadjoint operators in $L^{2}(R)$ defined by

$$
(p f)(t)=\frac{1}{i} \frac{d f}{d t}(t) \quad \text { and } \quad(q f)(t)=t f(t)
$$

for $f \in \mathscr{S}(\boldsymbol{R})$. Then $\mathscr{S}(\boldsymbol{R})$ is a core for both of $p$ and $q$. These operators satisfy 
the commutation relation $[p, q]=-i 1$ on $\mathscr{S}(\boldsymbol{R})$. Put

$$
a=2^{-1 / 2}(q+i p) \text { and } a^{+}=2^{-1 / 2}(q-i p) \text {. }
$$

Then these operators also satisfy the commutation relation $\left[a, a^{+}\right]=1$ on $\mathscr{S}(\boldsymbol{R})$. Let

$$
f_{n}(t)=\left(2^{n} n ! \pi^{1 / 2}\right)^{-1 / 2} H_{n}(t) \exp \left(-t^{2} / 2\right), \quad n \in Z_{+},
$$

where $H_{n}$ is the Hermite polynomial. Then $\left\{f_{n}: n \in Z_{+}\right\}$is an orthonormal basis of $L^{2}(R)$ and

$$
a^{+} f_{n}=(n+1)^{-1 / 2} f_{n+1} ; \quad \text { and } \quad a f_{n}=\left\{\begin{array}{cc}
n^{-1 / 2} f_{n-1} & (n \geq 1) \\
0 & (n=0) .
\end{array}\right.
$$

Thus $a^{+}$is a creation operator and $a$ is an annihilation operator. Since $a^{+}$ $\subset a^{*}$ and $a \subset\left(a^{+}\right)^{*}$, both $a$ and $a^{+}$are closable.

Now we define a Krein space $\{\Omega, J\}$ by

$$
\Re=L^{2}(R) \oplus L^{2}(R) \text { and } J=\left(\begin{array}{ll}
0 & 1 \\
1 & 0
\end{array}\right) .
$$

Then $h=i a^{*} \oplus(-i \bar{a})$ is a $\#$-selfadjoint operator. Using the multiplication operator $b:(b f)(t)=\exp \left(-t^{2} / 2\right) f(t)$ for $f \in L^{2}(R)$, we define a \#-unitary $v$ by $v=$ $b \oplus b^{-1}$. Then

$$
v h v^{\#}=2^{-1 / 2}(p \oplus p) \quad \text { on } \quad\left\{\mathscr{S}(\boldsymbol{R}) \cap L^{2}\left(\boldsymbol{R}, e^{t^{2}} d t\right)\right\} \oplus \mathscr{S}(\boldsymbol{R}) .
$$

Indeed, if $f \in \mathscr{S}(\boldsymbol{R})$ with $f \in L^{2}\left(\boldsymbol{R},\left(\exp t^{2}\right) d t\right)$, then

$$
\begin{aligned}
\left(b(i q+p) b^{-1} f\right)(t) & =i t f(t)+e^{-t^{2} / 2} \frac{1}{i} \frac{d}{d t}\left\{e^{t 2 / 2} f(t)\right\} \\
& =i t f(t)+e^{-t^{2} / 2} \frac{1}{i}\left\{t f(t)+f^{\prime}(t)\right\} e^{t^{2 / 2}} \\
& =i f^{\prime}(t)=(p f)(t)
\end{aligned}
$$

and if $g \in \mathscr{S}(\boldsymbol{R})$, then

$$
\begin{aligned}
\left(b^{-1}(-i q+p) b g\right)(t) & =-i \operatorname{tg}(t)+e^{t 2 / 2} \frac{1}{i} \frac{d}{d t}\left\{e^{-t 2 / 2} g(t)\right\} \\
& =-i \operatorname{tg}(t)+e^{t 2 / 2} \frac{1}{i}\left\{-\operatorname{tg}(t)+g^{\prime}(t)\right\} e^{-t^{2} / 2} \\
& =\frac{1}{i} g^{\prime}(t)=(p g)(t) .
\end{aligned}
$$

Moreover, 


$$
D\left(v^{*} v\right)=L^{2}(\boldsymbol{R}) \oplus\left\{L^{2}(\boldsymbol{R}) \cap L^{2}\left(\boldsymbol{R}, e^{2 t^{2}} d t\right)\right\},
$$

and hence

$$
\mathfrak{\Re}_{v}=L^{2}\left(\boldsymbol{R}, e^{-t^{2}} d t\right) \oplus L^{2}\left(\boldsymbol{R}, e^{t 2} d t\right) .
$$

It follows that $h \mid D\left(v^{*} v\right)$ is essentially selfadjoint in $\Re_{v}$. In this case, it is easy to see that $\operatorname{Sp}\left(\left\{v h v^{\sharp} \mid D_{0}\right\}^{-}\right)=\operatorname{Sp}\left(2^{-1 / 2} p\right)=R$ and $\operatorname{Sp}(-i \bar{a})=C$.

\title{
References
}

[1] T. Ando, Linear operators on Krein spaces, Mimeographed note, Hokkaido Univ., 1979.

[2] H. Araki, On a pathology in indefinite metric inner product space, Comm. Math. Phys., 85 (1982), 121-128.

[ 3 ] H. Araki, Indecomposable representations with invariant inner product-a theory of the Gupta-Bleuler triplet-, Preprint of RIMS, 1984.

[4] J. Bognár, Indefinite inner product spaces, Berlin, Heidelberg and New York, Springer-Verlag, 1974.

[ 5 ] I. Gohberg, P. Lancaster and L. Rodman, Matrices and indefinite scalar products, Basel, Boston and Stuttgart, Birkhäuser Verlag, 1983.

[ 6 ] I. S. Iohvidov, M. G. Krein and H. Langer, Introduction to the spectral theory of operators in spaces with indefinite metric, Berlin, Akademie-Verlag, 1982.

[7] T. Kato, Perturbation theory for linear operators, Berlin, Heidelberg and New York, Springer-Verlag, 1966.

[ 8 ] M. G. Krein, Introduction to the geometry of indefinite $J$-spaces and to the theory of operators in those spaces, Amer. Math. Soc. Transl., (2) 93 (1970), 103-176.

[9] M. A. Naimark, An analogue of Stone's theorem in a space with an indefinite metric, Soviet Math. Dokl., 7 (1966), 1366-1368.

[10] Y. Nakagami, Spectral analysis in Krein spaces, Sixth-seventh seminar on applied functional analysis, 1984, H. Umegaki, ed., 57-61.

[11] Y. Nakagami, Spectral theory on indefinite inner product spaces, Some topics on functional analysis, 1985, O. Takenouchi, ed., 51-82, (in Japanese).

[12] Y. Nakagami, Tomita's spectral analysis in Krein spaces, Publ. Res. Inst. Math. Sci., 22 (1986), 637-658.

[13] Y. Nakagami, On indefinite inner product spaces - \#-unitary operators in Krein spaces-, Bull. Yokohama City Univ., 37 \#2 (1986), 59-107, (in Japanese).

[14] N. Nakanishi, Indefinite metric quantum field theory, Progr. Theoret. Phys. Suppl., 51 (1972), 1-95.

[15] M. Tomita, Operators and operator algebras in Krein spaces, I - Spectral analysis in Pontrjagin spaces -, RIMS-koukyuuroku, 398 (1980), 131-158.

[16] M. Tomita, Spectral analysis in Krein spaces, The joint symposium of the 19th on real analysis and the 18th on functional analysis, 1980, 81-92, (in Japanese).

[17] K. Yosida, Functional analysis, Berlin, Heidelberg and New York, SpringerVerlag, 1968.

\author{
Yoshiomi Nakagami \\ DEPARTMENT OF MATHEMATiCS \\ YOKOHAMA CITY UNIVERSITY \\ 22-2 SETO, KANAZAWA-KU \\ YOKOHAMA, 236 JAPAN
}


and

Minoru TOMITA

Department of Applied Mathematics

FUKUOKA UNIVERSITY

8-19-1 NANAKUMA, JOHNAN-KU

FUKUOKA, 814-01 JAPAN 